\title{
Pyrrolodiazine derivatives as blue organic luminophores: synthesis and properties. Part $3^{\text {th }}$
}

\author{
Gheorghita N. Zbancioc ${ }^{a}$, Thomas Huhn ${ }^{\mathrm{b}}$, Ulrich Groth ${ }^{\mathrm{b}}$, Calin Deleanu ${ }^{\mathrm{c}}$, Ionel I. Mangalagiu ${ }^{\mathrm{a}, *}$ \\ 'Al 1. Cuza' University, Faculty of Chemistry, Organic Chemisry Department, Bd. Carol I no. 11, 700506 Iasi, Romania \\ - Universicy of Konstanz, Organic Chemistry Department, Fach M720, Universitätsstr. 10, D-78464 Konstanz, Germany \\ ''P. Poni' Institute of Macromolecular Chemistry, Aleea Grigore Ghica Voda $41+A$, RO-700487 lasi, Romania
}

Keywords:

Pyrrolodiazine

Fluorescence

Blue luminophores

Microwave

Cycloadditions

\begin{abstract}
A B S T R A C T
A fast efficient, general and environmentally friendly method for preparation of highly fluorescent derivatives containing the pyrrolodiazine moiety using microwave (MW) irradiation, in liquid phase, is reported. Under MW irradiation the yields are much higher, sometimes substantially (by almost double) and, the amount of solvent used is at least 5 -fold less. The pyrrolopyridazine (PP) derivatives are very intense blue emitters and have high quantum yields (up to 90\%) while pyrrolophthalazine (PHP) compounds are still intense blue emitters but the quantum yield is negligible. A certain influence of the substituents concerning fluorescence was found, those ones at the 7 position being crucial for fluorescence. The number of the substituents from the pyrrolo ring seems not to play an important role in regard with the fluorescence but, with an increasing number of substituents a certain hypsochromic shift in the absorption spectra was found.
\end{abstract}

\section{Introduction}

Synthesis of highly fluorescent derivatives with extended $\pi$-conjugation continues to arouse strong interest because of their applications as sensors and biosensors, electroluminescent materials, lasers, and other optoelectronic devices. ${ }^{14}$ Various classes and various strategies have been adopted to reach this goal. ${ }^{1.5}$ Fused $N$-heterocyclic rings offer very interesting optical properties. Pyrrolodiazine (PD) derivatives represent such a class (containing both a $\pi$-excessive pyrrole and a $\pi$-deficient diazine ring with one bridgehead nitrogen), being a 'pure' blue-emitting moiety. ${ }^{6,7}$ The absorption and fluorescence spectra of $\mathrm{N}$-heterocycles are solvent sensitive and depend, on one hand, on the nature of the substituents at the heterocycle, and on the other hand, on the positions of the substituents. Investigations on the synthesis of new blue luminous materials for applications in electroluminescent displays have attracted great attention, but there are very few single component deep blue- and pure red-emitting dyes. ${ }^{1,2}$ Because of the industrial demand, ${ }^{9}$ it is still essential to find molecules, which exhibit high fluorescence,

\footnotetext{
Ref. 7.

* Corresponding authar. Tel: +40 232 201343; fax: +40 232 201313; e-mail address: ionelm@uaicro (l.I. Mangalagiu).
}

little self-quenching, proper energy levels, pure RGB colour and high stability.

Microwave irradiation became a new trend in organic chemistry offering a versatile and facile pathway in a large variety of syntheses. ${ }^{10,11}$ So far, few studies have been reported regarding dipolar cycloaddition reactions of dazinium ylides and most of these have been conducted by our group. ${ }^{7.12}$

As a part of our work in the field of blue luminous materials for practical applications, ${ }^{7}$ we decided to study the relationship between optical properties and structure (the effect of substituents and conjugation), and to develop efficient, general and environmentally friendly methods for preparation of these derivatives using MW technologies.

\section{Results and discussion}

Considering the pyrrolopyridiazine (PP) moiety responsible for blue fluorescent properties, ${ }^{6,7}$ a rational design showed that the most suitable and accessible modification can be done on 3,4-position of the pyridazine (PY) heterocycle (expansion of the $\pi$ system conjugation with a benzene ring) and the 5-, 6- and 7-positions of the pyrrolo (PYR) ring (ester, amide or ketone substituents; with or without double bond in 4a,5- and 6,7-positions). In equal measure our interest was to study the influence of these modifications concerning the synthesis. 
<smiles></smiles>

The strategies adopted for construction of fluorescent PD derivatives $\mathrm{I}$, are depicted in Scheme 1. The preparation of all PD derivatives, $\mathbf{2}-\mathbf{5}$, involves two steps: initially $\mathrm{N}$-alkylation of the diazine [PY or phthalazine (PH)] followed by a $3+2$ dipolar cycloaddition of diazinium ylides $\mathbf{1}^{\prime}$ (generated in situ from the corresponding salts) to the corresponding dipolarophiles (activated alkenes or alkynes). tetrahydro-pyrrolodazine intermediate 4 , leading to more thermodynamically stable compounds $\mathbf{3 a}, \mathbf{b}$ and $\mathbf{5 a}-\mathbf{d}$. We may also notice that the saturated intermediates derived from acrylate (pathway iiib) have a greater tendency towards dehydrogenation, leading to a mixture of dihydropyrrolo-PY $(\mathbf{5 a}, \mathbf{b})$ and pyrrolo-PY (3a,b) while intermediates derived from acrylonitrile (pathway iiia) are leading to dihydropyrrolo-PY $(\mathbf{5 c}, \mathbf{d})$ only.

However, this strategy has some major disadvantages: longer reaction time (around $2-3 \mathrm{~h}$ ), lower yields (around $30 \%$ ), higher energy consumption and the need for large amounts of solvents, etc. This is why we decided to use MW technology, a non-conventional method, for syntheses. The MW assisted reactions were carried out using a monomode reactor, using a constant irradiation power and varying the temperature (the so-called power control'). The best results were obtained when we used $20 \%$ of the

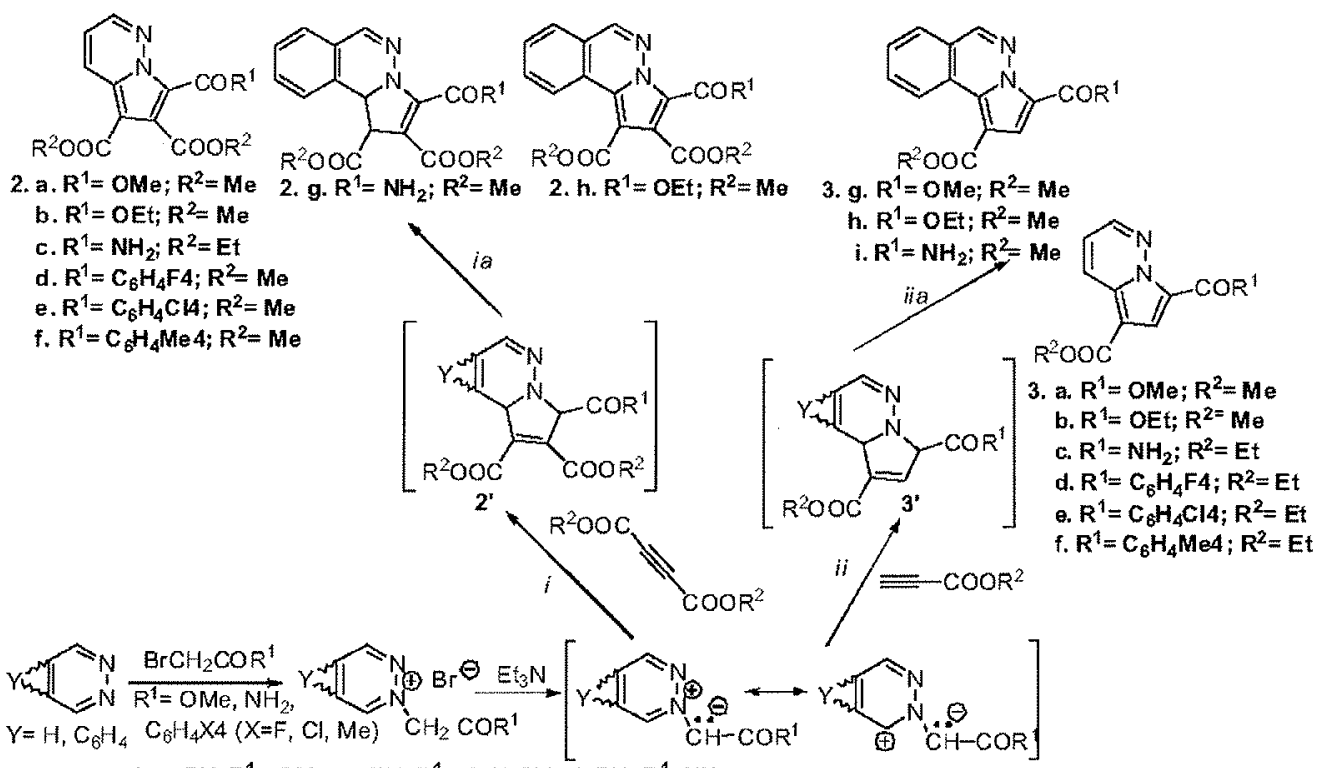

1. a. $P Y, R^{1}=O M e ;$ e.PY, $\mathrm{R}^{1}=\mathrm{C}_{6} \mathrm{H}_{4} \mathrm{Cl} 4 ; \mathrm{i}$. $\mathrm{PH}, \mathrm{R}^{1}=\mathrm{NH}_{2} 1$. b.PY, $R^{1}=$ OEt; f.PY, $R^{1}=C_{6} H_{4} M e 4 ;$ c. $P Y, R^{1}=N_{2} ;$ g. $P H, R^{1}=$ OMe; d. $P Y, R^{1}=C_{6} H_{4} F 4 ;$ h. $P H, R^{1}=O E t$<smiles>[Z]C1=C2C=CC=NN2C(C(=O)O)C1</smiles>
c. $\mathrm{R}^{1}=\mathrm{OMe}, \mathrm{Z}=\mathrm{CN}$ d. $R^{1}=O E t, Z=C N$
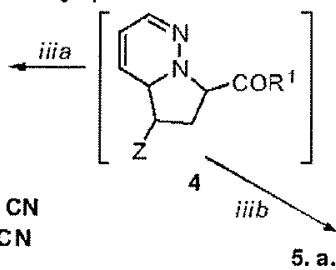

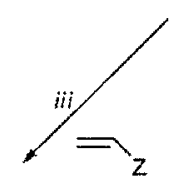

$\mathrm{Z}=\mathrm{CN}$; COOMe b. $R^{1}=O E f, Z=$ COOMe

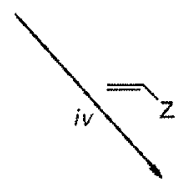<smiles>[Z]C1CC(C=O)N2N=CC=CC12</smiles>

4. b. $\mathrm{R}^{1}=\mathrm{NH}_{2}, \mathrm{Z}=\mathrm{CN}$ $\left[\mathrm{a} . \mathrm{R}^{1}=\mathrm{OMe}, \mathrm{Z}=\mathrm{CN}\right]$<smiles>[Z4]CC1c2ccccc2C=NN1C([Z20])CN</smiles>

4. $\mathbf{c} \cdot \mathrm{R}^{1}=\mathrm{NH}_{2}, \mathrm{Z}=\mathrm{CN}$

Scheme 1. Cyloaddition reactions of dazinium ylides with alkynes (pathways $i$ and ii) and alkenes (pathways iii and iv), under microwaves and classical heating conditions.

The reaction mechanism occurs as a typical Huisgen $[3+2]$ dipolar cycloaddition. When alkynes are used as dipolarophiles (pathways $\mathrm{i}$, ii), the PD moieties $\mathbf{2}$ and $\mathbf{3}$ are obtained. As intermediates are obtained the dihydropyrrolodazine derivatives $\mathbf{2}^{\prime}$ and 3 ', which have a greater tendency towards dehydrogenation leading to fully aromatised PDs, thermodynamically more stable (with the exception of compound $2 \mathrm{~g}$, which undergo an intramolecular rearrangement). In the case of alkenes (pathways iii, iv), the reactions occur differently according to the R-substituent and dipolarophiles structure, the first factor being determined. Thus, when $\mathrm{R}$ is an amide moiety the cycloaddition stops at the tetrahydro-pyrrolodazine $\mathbf{4 b}, \mathbf{c}$ stage. When $R$ is an ester moiety the cycloaddition is followed by an oxidative dehydrogenation of the full power of the magnetron $(800 \mathrm{~W})$. Table 1 lists optimised conditions we employed, under MW irradiation as well as under classical heating.

As indicated in Table 1, under MW heating the reaction times decrease dramatically (from several hours to $5 \mathrm{~min}$ ) and, the amount of solvent used is at least 5-fold less (see Experimental), so these reactions may be considered as environmentally friendly. Most remarkably the yields are higher with the use of MW heating, sometimes substantially (by almost double). We could also notice that the yields are higher when the substituent from the seventh position is an ester or amide group. A certain influence concerning yields between PY/PH heterocycle or between double/triple bound dipolarophiles is difficult to be determine. 
Table 1

Syntheses of PD derivatives under MW and classical heating conditions, in liquid phase

\begin{tabular}{|c|c|c|c|c|}
\hline \multirow[t]{2}{*}{ Compound } & \multicolumn{2}{|c|}{ Classical heating } & \multicolumn{2}{|l|}{ Microwaves } \\
\hline & $\begin{array}{l}\text { Reaction } \\
\text { time (min) }\end{array}$ & Yield (\%) & $\begin{array}{l}\text { Reaction } \\
\text { time (min) }\end{array}$ & Yield $(\%)$ \\
\hline $2 a\left(P Y, R^{1}=O M e ; R^{2}=M e\right)$ & 120 & 30 & 5 & 59 \\
\hline $2 \mathbf{b}\left(\mathrm{PY}, \mathrm{R}^{1}=\mathrm{OEt} ; \mathrm{R}^{2}=\mathrm{Me}\right)$ & 120 & 29 & 5 & 62 \\
\hline $2 c\left(P Y, R^{1}=N_{2} ; R^{2}=E t\right)$ & 120 & 32 & 5 & 51 \\
\hline $2 d\left(P Y, R^{1}=C_{6} H_{4} F 4 ; R^{2}=M e\right)$ & 120 & 10 & 5 & 14 \\
\hline $2 \mathrm{e}\left(\mathrm{PY}, \mathrm{R}^{1}=\mathrm{C}_{6} \mathrm{H}_{4} \mathrm{Cl} 4 \mathrm{R}^{2}=\mathrm{Me}\right)$ & 120 & 10 & 5 & 13 \\
\hline $2 \mathrm{f}\left(\mathrm{PY}, \mathrm{R}^{1}=\mathrm{C}_{6} \mathrm{H}_{4} \mathrm{Me4} ; \mathrm{R}^{2}=\mathrm{Me}\right)$ & 120 & 12 & 5 & 12 \\
\hline $\mathrm{Zg}\left(\mathrm{PH}_{1} \mathrm{R}^{3}=\mathrm{NH}_{2} ; \mathrm{R}^{2}=\mathrm{Me}\right)$ & 120 & 39 & 5 & 64 \\
\hline $\left.2 \mathbf{h}\left(\mathrm{PH}, \mathrm{R}^{1}=\mathrm{OE}\right) ; \mathrm{R}^{2}=\mathrm{Me}\right)$ & 120 & 36 & 5 & 61 \\
\hline $3 a\left(P Y, R^{1}=O M e ; R^{2}=M e\right)$ & 120 & 30 & 5 & 58 \\
\hline $3 \mathbf{b}\left(\mathrm{PY}, \mathrm{R}^{1}=\mathrm{OEt} ; \mathrm{R}^{2}=\mathrm{Me}\right)$ & 120 & 32 & 5 & 61 \\
\hline $3 c\left(P Y_{2} R^{1}=N_{2} ; R^{2}=E t\right)$ & 120 & 28 & 5 & 57 \\
\hline $3 d\left(P Y, R^{1}=C_{6} H_{4} F 4 ; R^{2}=E t\right)$ & 120 & 25 & 5 & 20 \\
\hline $3 e\left(P Y, R^{1}=\mathrm{C}_{6} \mathrm{H}_{4} \mathrm{Cl} 4 ; \mathrm{R}^{2}=\mathrm{Et}\right)$ & 120 & 83 & 5 & 87 \\
\hline $3 f\left(P Y, R^{1}=C_{6} H_{4} M e 4 ; R^{2}=E t\right)$ & 120 & 38 & 5 & 29 \\
\hline $3 \mathrm{~g}\left(\mathrm{PH}, \mathrm{R}^{1}=\mathrm{OMe} ; \mathrm{R}^{2}=\mathrm{Me}\right)$ & 120 & 41 & 5 & 66 \\
\hline 3h $\left(\mathrm{PH}, \mathbf{R}^{1}=\mathrm{OEt} ; \mathrm{R}^{2}=\mathrm{Me}\right)$ & 120 & 37 & 5 & 64 \\
\hline $3 \mathrm{i}\left(\mathrm{PH}, \mathrm{R}^{1}=\mathrm{NH}_{2} ; \mathrm{R}^{2}=\mathrm{Me}\right)$ & 120 & 40 & 5 & 68 \\
\hline $4 b\left(P Y, R^{1}=N_{2} ; Z=C N\right)$ & 180 & 31 & 5 & 52 \\
\hline $\mathrm{Ac}\left(\mathrm{PH}, \mathrm{R}^{1}=\mathrm{NH}_{2} ; \mathrm{Z}=\mathrm{CN}\right)$ & 180 & 29 & 5 & 53 \\
\hline $5 \mathrm{a}\left(\mathrm{PY}, \mathrm{R}^{1}=\mathrm{OMe} ; \mathrm{Z}=\mathrm{COOM}\right)$ & 180 & 23 & 5 & 48 \\
\hline $5 \mathbf{b}\left(\mathrm{PY}, \mathrm{R}^{1}=\mathrm{OEt} ; \mathrm{Z}=\mathrm{COOM}\right)$ & 180 & 23 & 5 & 49 \\
\hline $5 c\left(P Y, R^{1}=O M e ; Z=O N\right)$ & 180 & 36 & 5 & 62 \\
\hline $5 d\left(P Y, R^{1}=\mathrm{OEt} ; \mathrm{Z}=\mathrm{CN}\right)$ & 180 & 39 & 5 & 65 \\
\hline
\end{tabular}

In the next stage of our work, we studied the absorption and emission spectra of the obtained compounds. The spectra of all the compounds were recorded in ethanol, chloroform and cyclohexane solutions at room temperature. Relative quantum yields were determined by using anthracene in ethanol $\left(\phi=0.27\right.$ at $\left.25^{\circ} \mathrm{C}\right) .^{13}$ The studied PDs, although relatively similar in molecular structure, exhibit clear differences in their experimental absorption and emission spectra, as summarised in Table 2.

Table 2

$\lambda_{\max }(\mathrm{nm})$ of absorption spectra, fluorescence spectra and relative quantum yields (\%) of PD compounds $2-5$

\begin{tabular}{|c|c|c|c|c|c|c|}
\hline \multirow[t]{2}{*}{ Compound } & \multicolumn{3}{|c|}{$\begin{array}{l}\text { Fluorescence }\left(\lambda_{\max }, \mathrm{nm}\right) \\
\text { (quantum yields }(\%)\end{array}$} & \multicolumn{3}{|c|}{ Absorption $\left(\lambda_{\max }, \mathrm{nm}\right)$} \\
\hline & $\mathbf{E t O H}$ & $\mathrm{CHCl}_{3}$ & Gyclohexane & $\mathrm{EtOH}$ & $\mathrm{CHCl}_{3}$ & Cyclohexane \\
\hline $2 \mathrm{a}$ & $431(70)$ & $4,30(90)$ & $430(83)$ & 343 & 351 & 355 \\
\hline $2 b$ & $431(70)$ & $429(90)$ & $430(83)$ & 343 & 352 & 355 \\
\hline $2 c$ & $429(63)$ & $431(82)$ & $434(76)$ & 356 & 362 & 366 \\
\hline $2 d$ & $446(1)$ & $436(8)$ & $433(11)$ & 334 & 335 & 329 \\
\hline $2 e$ & $448(2)$ & $439(3)$ & $434(-)$ & 334 & 334 & 329 \\
\hline $2 f$ & $451(1)$ & $438(7)$ & $436(10)$ & 332 & 333 & 327 \\
\hline $2 \mathrm{~g}$ & $487(1)$ & $471(1)$ & Insoluble & 374 & 375 & Insoluble \\
\hline $2 \mathrm{~h}$ & $448(4)$ & $431(5)$ & $434(8)$ & 314 & 319 & 322 \\
\hline $3 \mathbf{a}$ & $430(66)$ & $423(91)$ & $416(85)$ & 349 & 357 & 361 \\
\hline $3 b$ & $429(66)$ & $422(91)$ & $416(85)$ & 349 & 358 & 361 \\
\hline $3 c$ & $436(89)$ & $437(76)$ & Insoluble & 365 & 370 & Insoluble \\
\hline 3d & $442(1)$ & $436(5)$ & Insoluble & 333 & 335 & Insoluble \\
\hline $3 e$ & $430(2)$ & $436(7)$ & Insoluble & 330 & 332 & Insoluble \\
\hline $3 \mathbf{f}$ & $433(1)$ & $437(4)$ & $432(8)$ & 333 & 334 & 329 \\
\hline 3g & $446(4)$ & $430(5)$ & $432(9)$ & 315 & 319 & 322 \\
\hline 3h & $446(4)$ & $430(4)$ & $432(8)$ & 316 & 320 & 322 \\
\hline $3 \mathbf{i}$ & $448(3)$ & $432(2)$ & Insoluble & 319 & 322 & Insoluble \\
\hline $4 b$ & $442(2)$ & $439(2)$ & Insoluble & 326 & 321 & Insoluble \\
\hline $4 c$ & $427(1)$ & $439(5)$ & Insoluble & 316 & 304 & Insoluble \\
\hline $5 \mathbf{a}$ & $430(9)$ & $427(18)$ & $415(40)$ & 470 & 479 & 496 \\
\hline $5 \mathbf{b}$ & $430(9)$ & $427(18)$ & $415(40)$ & 470 & 479 & 496 \\
\hline $5 c$ & $432(3)$ & $424(4)$ & $420(4)$ & 485 & 488 & 508 \\
\hline $5 d$ & $433(4)$ & $424(4)$ & $419(4)$ & 484 & 488 & 508 \\
\hline
\end{tabular}

As expected, conjugation is determined concerning fluorescence and quantum yields. As shown in Table 2, fully aromatised and conjugated pyrrolo-PY $(\mathbf{2 a}-\mathbf{c}$ and $\mathbf{3 a - c})$ are very intense blue emitters ( $\lambda_{\max }$ of fluorescence around $415-435 \mathrm{~nm}, \lambda_{\max }$ of absorption around $340-370 \mathrm{~nm}$ ) and extremely high quantum yield

(up to 90\%), partially saturated dihydropyrrolo-PY $(\mathbf{5 a}-\mathbf{d})$ are redshifted $\left(\lambda_{\max }\right.$ of fluorescence around $425-435 \mathrm{~nm}, \lambda_{\max }$ of absorption around $470-510 \mathrm{~nm}$ ) and have a low quantum yield (around $5-40 \%)$, while tetrahydro-pyrrolodiazine $(4 \mathbf{b}, \mathbf{c})$ have a negligible quantum yield (les than $5 \%$ ), the fluorescence are even more redshifted $\left(\lambda_{\max }\right.$ of fluorescence around $427-442 \mathrm{~nm}$ ) and, intriguing, the absorption if blue-shifted $\left(\lambda_{\max }\right.$ of absorption around 304-326 nm), Scheme 2 .<smiles>c1ccc2c(c1)cnn1cccc21</smiles>

However, fully aromatised pyrrolo-PH (2h and $3 \mathbf{g}-\mathbf{i})$ have an unexpected behaviour. Even so they are very intense blue emitters ( $\lambda_{\max }$ of fluorescence around $430-448 \mathrm{~nm}$ ), they have a negligible quantum yield (less than 10\%) and an unusual blue-shifted absorption $\left(\lambda_{\max }\right.$ of absorption around $\left.314-322 \mathrm{~nm}\right)$. This is very unusual because normally extension of conjugation should have an opposite effect: a red-shift in absorption and increasing of quantum yield. ${ }^{1}$ We presume that this is a question of aromaticity mostly ( $\pi$-stacking interactions, favourable or unfavourable, could play a role). Unless the pyrrolo-PH have a more extended conjugated system (three rings compared with pyrrolo-PY with only two), the pyrrolopyridazine unit is more delocalised in pyrrolo-PY compounds (resonance structure I). The resonance structure II will have a major contribution to the structure of pyrrolo-PH, due to the strong tendency of the fused benzene ring to remain aromatic. Consequently, the delocalisation in pyrrolo-PH compounds will decrease and they will appear blue-shifted in absorption spectra. Similar consideration has been published for related cases. ${ }^{6}$

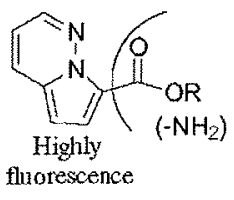

The data from Table 2 indicates also a certain influence of the substituents, those ones from position 7 being crucial for fluorescence.

When the substituent is an ester or amide group the pyrrolo-PY compounds have an intense blue fluorescence and a very high quantum yield. When the substituent is a ketone the pyrrolo-PY compounds still are blue emitters (red-shifted, $\lambda_{\mathrm{max}}$ of fluorescence around $430-450 \mathrm{~nm}$ ) but the quantum yield is negligible. Again, a feasible explanation should be related to the conjugation: pyrrolo-PY compounds bearing esters (amide, respectively) in position 7 have a stronger conjugation compared with those ones bearing a keto-moiety.

The number of the substituents from the pyrrolo ring doesn't seem to play an important role with regard to the fluorescence properties. Data from Table 2 indicates that both classes of compounds, with three or two substituents (e.g., $\mathbf{2 a - c}$ vs $3 \mathbf{a}-\mathbf{c}$ ), have almost the same fluorescent properties (very blue) and quantum yield (around 90\%). However, we may notice in the absorption, a certain hypsochromic shift with the increasing of the number of substituents $\left[\lambda_{\max }\right.$ absorption around $343-366 \mathrm{~nm}$ (for three substituents) and around $349-370 \mathrm{~nm}$ (for two substituents)].

\section{Conclusions}

We report herein a fast, efficient and straightforward method for MW preparation of highly fluorescent derivatives containing the PD 
<smiles>c1ccc2c(c1)cnn1cccc21</smiles>

D

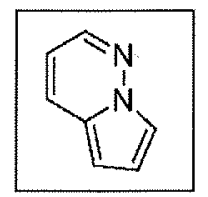

A<smiles>C1=CC2=CCCN2N=C1</smiles>

B

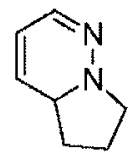

C

$\frac{\text { Fluorescence red-shifted, }}{\text { quantum yield decreasing }} \longrightarrow$

Scheme 2. Futrescence and quantum yield variation in the pyrolodiazine series.

moiety. Remarkably, under MW irradiation the yields are much higher, sometimes substantially (by almost double) and, the amount of solvent used is at least 5-fold less. Presence of an ester or amide group in the position 7 of the pyrrolopyridazine skeleton increases the chemical yield. The fully aromatised pyrrolo[1,2-b]pyridazines are very intense blue emitters and have high quantum yields while pyrrolophthalazine compounds are still intense blue emitters but the quantum yield is negligible. A feasible explication for this behaviour is presented. A certain influence of the substituents concerning fluorescence was found, those one at the position 7 being crucial for fluorescence. Again, feasible explications for this behaviour are presented. The number of the substituents from the pyrrolo ring doesn't seem to play an important role regarding fluorescence but, with the increase of the number of substituents a certain hypsochromic shift in the absorption spectra was found.

\section{Experimental section}

\subsection{General procedure}

All reagents and solvents employed were of the best grade available and were used without further purification. The ${ }^{1} \mathrm{H}$ and ${ }^{13} \mathrm{CNMR}$ spectra and two-dimensional experiments 2D-COSY, 2D-HETCOR (HMQC), long range 2D-HETCOR (HMBC) were recorded on a Bruker Avance $400 \mathrm{DRX}$ spectrometer at $400 / 100 \mathrm{MHz}$. Chemical shifts are given in parts per million ( $\delta$-scale), coupling constants $(\delta)$ in hertz and downfield shift from internal tetramethylsilane $(\hat{\delta} 0.00 \mathrm{ppm})$. The IR spectra were recorded on an FT-IR Shimadzu Prestige 8400s spectrophotometer in $\mathrm{KBr}$. UV-vis spectra were recorded on a Shimadzu $1800 \mathrm{PC}$ spectrophotometer in ethanol, chloroform, cyclohexane (spectroscopic grade) solution. Fluorescence measurements were performed on a Perkin-Elmer LS 50 fluorescence spectrophotometer, in the same solvents as for the UV-vis spectra. For the microwave irradiation we used an $800 \mathrm{~W}$ STAR SYSTEM-2 monomode reactor (CEM Corporation). Melting points were determined using an electrothermal apparatus and are uncorrected. Flash chromatography was performed with Aldrich $230-400$ mesh silica gel. TLC was carried out on Merck silica gel 60-F-254 plates. Compounds $\mathbf{2 a}, \mathbf{2 b}$ and $\mathbf{2 h}$, were initially investigated by Wudl et al.; ${ }^{6}$ here we obtained these compounds classical by a modified pathway and also, using a new method, under MW. All the remaining compounds are new being synthetized by us. Some spectral data of compounds $\mathbf{3 a}, \mathbf{b}$ and $\mathbf{5 a}-\mathbf{d}$ were initially published by us in a Short Communication. ${ }^{\text {7a }}$

\subsection{Experimental procedure for $[3+2]$ dipolar cycloaddition under MW classical and heating}

4.2.1. 5,6,7-Tri-(methoxycarbonyl)pyrrolol 1,2-bjpyridazine (2a). A mixture of cycloimmonium salt $1 \mathrm{a}(1.17 \mathrm{~g}, 5 \mathrm{mmol})$ and dimethyl acetylenedicarboxylate $(0.68 \mathrm{~mL}, 5.5 \mathrm{mmol})$ was suspended in anhydrous benzene, $40 \mathrm{~mL}$ under classical heating or $10 \mathrm{~mL}$ under MW irradiation. Then, triethylamine $(0.77 \mathrm{~mL}, 5.5 \mathrm{mmol})$ was added. Under classical conditions, the solution was refluxed (oil bath) for $2 \mathrm{~h}$. Under microwave heating, the solution was exposed to microwave for $5 \mathrm{~min}$. Using MW irradiation, the best results were obtained using a constant irradiation power ( $20 \%$ from the full power of the magnetron, $800 \mathrm{~W}$ ) and varying the temperature (the so-called 'power control'). The resulting mixture was filtered hot to remove triethylamine hydrobromide and the clear solution was evaporated in vacuo to give the crude product, which was purified by flash chromatography $\left(99 / 1 \mathrm{CH}_{2} \mathrm{Cl}_{2} / \mathrm{CH}_{3} \mathrm{OH}\right)$ to give $5,6,7-t r i-$ (methoxycarbonyl)pyrrolo[1,2-b]pyridazine $2 \mathrm{a}(0.44 \mathrm{~g}$, 30\% (under classical heating) and $0.86 \mathrm{~g}, 59 \%$ (under microwaves)) as a white solid, mp 168-169 ${ }^{\circ} \mathrm{C}$. Found: $\mathrm{C}, 53.32 ; \mathrm{H}, 4.06 ; \mathrm{N}, 9.48 . \mathrm{C}_{13} \mathrm{H}_{12} \mathrm{~N}_{2} \mathrm{O}_{6}$ (292) requires $\mathrm{C}, 53.43 ; \mathrm{H}, 4.14 ; \mathrm{N}, 9.59 \% ; R_{f}\left(99 / 1 \mathrm{CH}_{2} \mathrm{Cl}_{2} / \mathrm{CH}_{3} \mathrm{OH}\right)$ 0.21 ; IR ( $\mathrm{KBr}, \mathrm{cm}^{1}$ ): 3096 (C-H arom.), 2950 (C-H aliph.), 1725, $1722,1709(\mathrm{C}=\mathrm{O}$ est $), 1600,1560,1505,1460(\mathrm{C}=\mathrm{C}, \mathrm{C}=\mathrm{N}), 1230$, $1130(\mathrm{C}-\mathrm{O}-\mathrm{C}) ;{ }^{1} \mathrm{H} \mathrm{NMR}\left(\mathrm{CDCl}_{3}, \delta, \mathrm{ppm}, J, \mathrm{~Hz}\right): 3.92\left(\mathrm{~s}, 3 \mathrm{H}: \mathrm{CH}_{3}\right.$ from 5 position), 3.96 ( $\mathrm{s}, 3 \mathrm{H}: \mathrm{CH}_{3}$ from 7 position), 4.02 ( $\mathrm{s}, 3 \mathrm{H}: \mathrm{CH}_{3}$ from 6 position), 7.16 (dd, $J=9.2,4.4,1 \mathrm{H}: \mathrm{H}_{3}$ ), 8.56 (dd, $J=4.4,1.8,1 \mathrm{H}: \mathrm{H}_{4}$ ), 8.64 (dd, $J=9.2,1.8,1 \mathrm{H}: \mathrm{H}_{2}$ ); ${ }^{13} \mathrm{C}$ NMR (TMS, $\mathrm{CDCl}_{3}, \delta, \mathrm{ppm}$ ): 52.0 $\left(\mathrm{CH}_{3}\right.$ from 5 position, $\left.\mathrm{COOMe}\right), 52.3\left(\mathrm{CH}_{3}\right.$ from 7 position, $\left.\mathrm{COOMe}\right)$, $53.1\left(\mathrm{CH}_{3}\right.$ from 6 position, COOMe), $102.8\left(\mathrm{C}_{4 \mathrm{a}}\right), 117.11\left(\mathrm{C}_{5}\right), 117.6$ $\left(C_{3}\right), 128.4\left(C_{2}\right), 128.8\left(C_{6}\right), 131.9\left(C_{7}\right), 145.1\left(C_{4}\right), 158.7$ (CO from 7 position), 162.7 (CO from 5 position), 165.6 (CO from 6 position); MS (EI, $m / z): 292\left(\mathrm{M}^{+}, 78 \%\right), 261$ (P.B., 100\%), $231(10.71 \%), 203(18 \%)_{2}$ $189(9.5 \%), 144(9.8 \%), 88(4.9 \%)$.

4.2.2. 7-Ethoxy-5,6-di-(methoxycarbonyl)pyrrolo[ 1,2-bjpyridazine (2b). A mixture of cycloimmonium salt $1 \mathrm{~b}(1.24 \mathrm{~g}, 5 \mathrm{mmol})$ and dimethyl acetylenedicarboxylate $(0.68 \mathrm{~mL}, 5.5 \mathrm{mmol})$ was suspended in anhydrous benzene, $40 \mathrm{~mL}$ under classical heating or $10 \mathrm{~mL}$ under $\mathrm{MW}$ irradiation. Then, triethylamine $0.77 \mathrm{~mL}$, $5.5 \mathrm{mmol}$ ) was added. Under classical conditions, the solution was refluxed (oil bath) for $2 \mathrm{~h}$. Under microwave heating, the solution was exposed to microwave for $5 \mathrm{~min}$. Using MW irradiation, the best results were obtained using a constant irradiation power ( $20 \%$ from the full power of the magnetron, $800 \mathrm{~W}$ ) and varying the temperature (the so-called 'power control'). The resulting mixture was filtered hot to remove triethylamine hydrobromide and the clear solution was evaporated in vacuo to give the crude product, which was purified by flash chromatography $\left(99 / 1 \mathrm{CH}_{2} \mathrm{Cl}_{2} / \mathrm{CH}_{3} \mathrm{OH}\right)$ to give 7-ethoxy-5,6-di-(methoxycarbonyl)pyrrolo[ 1,2-b]pyridazine $2 \mathrm{~b}(0.44 \mathrm{~g}, 29 \%$ (under classical heating) and $0.95 \mathrm{~g}, 62 \%$ (under microwaves) ) as a white solid, $\mathrm{mp} 137-138^{\circ} \mathrm{C}$. Found: $\mathrm{C}, 54.78 ; \mathrm{H}$, 4.52; $\mathrm{N}, 9.01 . \mathrm{C}_{14} \mathrm{H}_{14} \mathrm{~N}_{2} \mathrm{O}_{6}(306)$ requires $\mathrm{C}, 54.90 ; \mathrm{H}, 4.61 ; \mathrm{N}, 9.15 \%$; $R_{f}\left(99 / 1 \mathrm{CH}_{2} \mathrm{Cl}_{2} / \mathrm{CH}_{3} \mathrm{OH}\right) 0.18$; IR $\left(\mathrm{KBr}, \mathrm{cm}^{1}\right): 3112$ (C-H arom.), $2956(\mathrm{C}-\mathrm{H}$ aliph. $), 1751,1712,1677(\mathrm{C}=\mathrm{O}$ est. $), 1618,1502,1448$, $1406(\mathrm{C}=\mathrm{C}, \mathrm{C}=\mathrm{N}), 1284,1127(\mathrm{C}-\mathrm{O}-\mathrm{C}) ;{ }^{1} \mathrm{H} \mathrm{NMR}\left(\mathrm{CDCl}_{3}, \delta, \mathrm{ppm}, j\right.$, $\mathrm{Hz}): 1.36\left(\mathrm{t}, J=6.8,3 \mathrm{H}: \mathrm{CH}_{3}\right.$ from 7 position), $3.89\left(\mathrm{~s}, 3 \mathrm{H}: \mathrm{CH}_{3}\right.$ from 5 position), 3.98 ( $\mathrm{s}, 3 \mathrm{H}: \mathrm{CH}_{3}$ from 6 position), 4.39 (q, $J=6.8,2 \mathrm{H}: \mathrm{CH}_{2}$ from 7 position), 7.13 (dd, $\left.J=8.8,4.0,1 \mathrm{H}: \mathrm{H}_{3}\right), 8.54\left(\mathrm{~d}, J=8.8,1 \mathrm{H}: \mathrm{H}_{4}\right.$ ), $8.60\left(\mathrm{~d}, j=4.0,1 \mathrm{H}: \mathrm{H}_{2}\right):{ }^{13} \mathrm{C}$ NMR (TMS, $\left.\mathrm{CDCl}_{3}, \delta_{1} \mathrm{ppm}\right): 14.0\left(\mathrm{CH}_{3}\right.$ from 7 position, COOEt), $51.9\left(\mathrm{CH}_{3}\right.$ from 5 position, COOMe), 52.8 $\left(\mathrm{CH}_{3}\right.$ from 6 position, $\left.\mathrm{COOMe}\right), 61.2\left(\mathrm{CH}_{2}\right.$ from 7 position, COOEt), $102.6\left(C_{4 a}\right), 117.1\left(C_{3}\right), 117.5\left(C_{5}\right), 128.3\left(C_{6}\right), 128.8\left(C_{2}\right), 131.7\left(C_{7}\right)$, $145.0\left(\mathrm{C}_{4}\right), 158.0$ (CO from 7 position), 162.7 (CO from 5 position), 165.5 (CO from 6 position); MS (EI, m/z): $304\left(\mathrm{M}^{+}, 90.8 \%\right), 275$ 
(24.6\%), 261 (47\%), 234 (P.B.; 100\%), 203 (88.7\%), 144 (19.7\%), 117 $(4.2 \%), 144(9.8 \%), 76(2.8 \%)$.

4.2.3. Diethy! 7-carbamoylpyrrolol1,2-bjpyridazine-5,6-dicarboxylate (2c). A mixture of cycloimmonium salt 1c $(1.09 \mathrm{~g}, 5 \mathrm{mmol})$ and diethyl acetylenedicarboxylate $(0.88 \mathrm{~mL}, 5.5 \mathrm{mmol})$ was suspended in anhydrous benzene, $40 \mathrm{~mL}$ under classical heating or $10 \mathrm{~mL}$ under MWirradiation. Then, triethylamine $(0.77 \mathrm{~mL}, 5.5 \mathrm{mmol})$ was added. Under classical conditions, the solution was refluxed (oil bath) for $2 \mathrm{~h}$. Under microwave heating, the solution was exposed to microwave for 5 min. Using MW irradiation, the best results were obtained using a constant irradiation power ( $20 \%$ from the full power of the magnetron, $800 \mathrm{~W}$ ) and varying the temperature (the so-called 'power control'). The resulting mixture was filtered hot to remove triethylamine hydrobromide and the clear solution was evaporated in vacuo to give the crude product, which was purified by flash chromatography $\left(99 / 1 \mathrm{CH}_{2} \mathrm{Cl}_{2} / \mathrm{CH}_{3} \mathrm{OH}\right)$ to give the diethyl 7-carbamoylpyrrolo [1,2-b]pyridazine-5,6-dicarboxylate $2 \mathrm{c}(0.49 \mathrm{~g}, 32 \%$ (under classical heating) and $0.78 \mathrm{~g}, 51 \%$ (under microwaves) ) as a white solid, mp 196-197 ${ }^{\circ} \mathrm{C}$ Found: $\mathrm{C}, 55.07 ; \mathrm{H}, 4.91 ; \mathrm{N}, 13.71 . \mathrm{C}_{14} \mathrm{H}_{15} \mathrm{~N}_{3} \mathrm{O}_{5}$ (305) requires $\mathrm{C}_{\mathrm{t}} 55.08 ; \mathrm{H}, 4.95 ; \mathrm{N}, 13.76 \% ; R_{f}\left(99 / 1 \mathrm{CH}_{2} \mathrm{Cl}_{2} / \mathrm{CH}_{3} \mathrm{OH}\right) 0.17 ; \mathrm{IR}$ $\left(\mathrm{KBr}, \mathrm{cm}^{1}\right): 3446$ ( $\mathrm{N}-\mathrm{H}$ amide), 3068 (C-H arom) $2943(\mathrm{C}-\mathrm{H}$

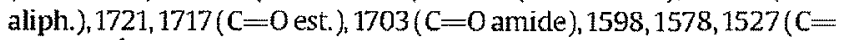
Carom.); $1 \mathrm{HNMR}\left(\mathrm{CDCl}_{3}, \delta, \mathrm{ppm}, J, \mathrm{~Hz}\right): 1.41-1.37\left(\mathrm{~m}, 6 \mathrm{H}: 2 \times \mathrm{CH}_{3}\right.$ from 5 and 6 position), $4.36\left(q, j=7.2,2 \mathrm{H}: \mathrm{CH}_{2}\right.$ from 5 position), 4.42 ( $q$, $J=7.2,2 \mathrm{H}: \mathrm{CH}_{2}$ from 6 position), 7.12 (br $\left.s, 1 \mathrm{H}: \mathrm{NH}\right), 7.42(\mathrm{q}, J=4.8,9.2$, $\left.1 \mathrm{H}: \mathrm{H}_{3}\right), 8.50$ (br s, $\left.1 \mathrm{H}: \mathrm{NH}\right), 8.73\left(\mathrm{dd}, J=1.6,4.8,1 \mathrm{H}: \mathrm{H}_{4}\right), 8.76(\mathrm{dd}, J=1.6$, 9.2,1H: $\left.\mathrm{H}_{2}\right) ;{ }^{13} \mathrm{CNMR}$ (TMS, $\left.\mathrm{CDCl}_{3} \delta, \mathrm{ppm}\right): 15.1\left(\mathrm{CH}_{3}\right.$ from 6 position), $15.3\left(\mathrm{CH}_{3}\right.$ from 5 position), 61.8 ( $\mathrm{CH}_{2}$ from 6 position), $62.4\left(\mathrm{CH}_{2}\right.$ from 5 position $), 104.3\left(C_{5}\right), 106.3\left(C_{6}\right), 118.7\left(C_{3}\right), 124.3\left(C_{7}\right), 130.7\left(C_{4}\right), 130.7$ $\left(C_{4 a}\right), 146.3\left(C_{2}\right), 159.2$ (CO from 7 position), 161.9 (CO from 5 position), 166.8 (CO from 6 position).

4.2.4. Dimethyl 7-(4-fluorobenzoyl)pyrrolol 1,2-blpyridazine-5,6-dicarboxylate (2d). A mixture of cycloimmonium salt $1 \mathrm{~d}(1.49 \mathrm{~g}$. $5 \mathrm{mmol})$ and dimethyl acetylenedicarboxylate $(0.68 \mathrm{~mL}, 5.5 \mathrm{mmol})$ was suspended in anhydrous benzene, $40 \mathrm{~mL}$ under classical heating or $10 \mathrm{~mL}$ under $\mathrm{MW}$ irradiation. Then, triethylamine $10.77 \mathrm{~mL}$, $5.5 \mathrm{mmol}$ ) was added. Under classical conditions, the solution was refluxed (oil bath) for $2 \mathrm{~h}$. Under microwave heating, the solution was exposed to microwave for 5 min. Using MW irradiation, the best results were obtained using a constant irradiation power ( $20 \%$ from the full power of the magnetron, $800 \mathrm{~W}$ ) and varying the temperature (the so-called 'power control'). The resulting mixture was filtered hot to remove triethylamine hydrobromide and the clear solution was evaporated in vacuo to give the crude product, which was purified by flash chromatography $\left(99 / 1 \mathrm{CH}_{2} \mathrm{Cl}_{2} / \mathrm{CH}_{3} \mathrm{OH}\right)$ to give the dimethyl 7-(4fluorobenzoyl)pyrrolo[1,2-bjpyridazine -5,6-dicarboxylate $2 \mathrm{~d}(0.18 \mathrm{~g}$, $10 \%$ (under classical heating) and $0.25 \mathrm{~g}, 14 \%$ (under microwaves) as a white solid, mp $156-158^{\circ} \mathrm{C}$. Found: C, 60.66; H, 3.61; N, 7.77. $\mathrm{C}_{18} \mathrm{H}_{13} \mathrm{FN}_{2} \mathrm{O}_{5}$ (356) requires $\mathrm{C}, 60.68 ; \mathrm{H}_{1} 3.68 ; \mathrm{N}, 7.86 \% ; R_{f}(99 / 1$ $\left.\mathrm{CH}_{2} \mathrm{Cl}_{2} / \mathrm{CH}_{3} \mathrm{OH}\right) 0.32 ; \mathrm{IR}\left(\mathrm{KBr}_{1} \mathrm{~cm}^{1}\right): 3070(\mathrm{C}-\mathrm{H}$ arom), $2956(\mathrm{C}-\mathrm{H}$ aliph.), 1739, 1704( $\mathrm{C}=$ Oest.), 1643 ( $\mathrm{C}=$ O keto $), 1604,1537,1504,1452$ $(\mathrm{C}=\mathrm{C}, \mathrm{C}=\mathrm{N}), 1247,1132(\mathrm{C}-\mathrm{O}-\mathrm{C}),{ }^{1} \mathrm{H}$ NMR $\left(\mathrm{CDCl}_{3}, \hat{\delta}, \mathrm{ppm}, J, \mathrm{~Hz}\right): 3.65$ ( $\mathrm{s}, 3 \mathrm{H}: \mathrm{CH}_{3}$ from 5 position), 3.92 ( $\mathrm{s}, 3 \mathrm{H}: \mathrm{CH}_{3}$ from 6 position), 7.08 (dd, $\left.J=4.8,9.2,1 \mathrm{H}: \mathrm{H}_{3}\right), 7.13\left(\mathrm{dd}, J=8.4,8.8,2 \mathrm{H}: \mathrm{H}_{11}\right), 7.82(\mathrm{dd}, J=8.4,5.2,2 \mathrm{H}:$ $\left.\mathrm{H}_{10}\right), 8.32\left(\mathrm{dd}, J=4.8,1 \mathrm{H}: \mathrm{H}_{4}\right), 8.62\left(\mathrm{dd}, J=9.2,1 \mathrm{H}: \mathrm{H}_{2}\right) ;{ }^{13} \mathrm{CNMR}$ (TMS, $\left.\mathrm{CDCl}_{3}, \delta, \mathrm{ppm}\right): 51.9\left(\mathrm{CH}_{3}\right.$ from 5 position), $52.6\left(\mathrm{CH}_{3}\right.$ from 6 position), $103.2\left(C_{5}\right), 115.7,115.5\left(\mathrm{~d}, J=22, C_{11}\right), 117.3\left(C_{3}\right), 126.0\left(C_{6}\right), 128.8\left(C_{4}\right)$, $130.9\left(C_{7}\right), 132.1,132.0\left(\mathrm{~d}, J=10, C_{10}\right), 132.1\left(C_{4 a}\right), 138.4\left(C_{12}\right), 144.7\left(C_{2}\right)$, 162.9 (CO from 5 position), 164.5 (CO from 6 position), $184.0\left(C_{8}\right.$, keto).

4.2.5. Dimethyl 7-(4-chlorobenzoyl)pyrrolo[1,2-b]pyridazine-5,6-dicarboxylate (2e). A mixture of cycloimmonium salt $1 \mathrm{e}(1.57 \mathrm{~g}$, $5 \mathrm{mmol}$ ) and dimethyl acetylenedicarboxylate $(0.68 \mathrm{~mL}, 5.5 \mathrm{mmol})$ was suspended in anhydrous benzene, $40 \mathrm{~mL}$ under classical heating or $10 \mathrm{~mL}$ under MW irradiation. Then, triethylamine $(0.77 \mathrm{~mL}, 5.5 \mathrm{mmol})$ was added. Under classical conditions, the solution was refluxed (oil bath) for $2 \mathrm{~h}$. Under microwave heating, the solution was exposed to microwave for $5 \mathrm{~min}$. Using MW irradiation, the best results were obtained using a constant irradiation power ( $20 \%$ from the full power of the magnetron, $800 \mathrm{~W})$ and varying the temperature (the so-called 'power control'). The resulting mixture was filtered hot to remove triethylamine hydrobromide and the clear solution was evaporated in vacuo to give the crude product, which was purified by flash chromatography (99/1 $\mathrm{CH}_{2} \mathrm{Cl}_{2} / \mathrm{CH}_{3} \mathrm{OH}$ ) to give the dimethyl 7-(4-chlorobenzoyl)pyrrolo[ $1,2-$ bjpyridazine-5,6-dicarboxylate $2 \mathrm{e}(0.19 \mathrm{~g}, 10 \%$ (under classical heating) and $0.24 \mathrm{~g}, 13 \%$ (under microwaves)) as a white solid, mp $178-179{ }^{\circ} \mathrm{C}$. Found: $\mathrm{C}, 57.98 ; \mathrm{H}, 3.47 ; \mathrm{N}, 7.45 . \mathrm{C}_{18} \mathrm{H}_{13} \mathrm{ClN}_{2} \mathrm{O}_{5}(372)$ requires $\mathrm{C}, 58.00 ; \mathrm{H}, 3.52 ; \mathrm{N}, 7.52 \% ; \mathrm{R}_{f}\left(99 / 1 \mathrm{CH}_{2} \mathrm{Cl}_{2} / \mathrm{CH}_{3} \mathrm{OH}\right) 0.30 ; \mathrm{IR}$ $\left(\mathrm{KBr}, \mathrm{cm}^{1}\right.$ ): 3091 (C-H arom.), 2950 (C-H aliph.), 1744, 1707 (C=O est.), $1648(C=$ o keto $), 1588,1500,1452,1389(C=C, C=N), 1242$, $1106(\mathrm{C}-\mathrm{O}-\mathrm{C}) ;{ }^{1} \mathrm{H} \mathrm{NMR}\left(\mathrm{CDCl}_{3}, \delta, \mathrm{ppm}_{1}, \mathrm{~Hz}\right): 3.66\left(\mathrm{~s}, 3 \mathrm{H}: \mathrm{CH}_{3}\right.$ from 5 position), $3.92\left(\mathrm{~s}, 3 \mathrm{H}: \mathrm{CH}_{3}\right.$ from 6 position), $7.10\left(\mathrm{dd}_{1} j=3.2,8.8,1 \mathrm{H}\right.$ : $\left.\mathrm{H}_{3}\right), 7.43\left(\mathrm{~d}, J=8.4,2 \mathrm{H}: \mathrm{H}_{11}\right), 7.73\left(\mathrm{~d}, J=8.4,2 \mathrm{H}: \mathrm{H}_{10}\right), 8.33(\mathrm{~d}, J=3.2$, 1H: $\left.\mathrm{H}_{4}\right), 8.61\left(\mathrm{~d}, J=8.8,1 \mathrm{H}: \mathrm{H}_{2}\right):{ }^{13} \mathrm{C}$ NMR (TMS, $\left.\mathrm{CDCl}_{3}, \delta, \mathrm{ppm}\right): 52.0$ ( $\mathrm{CH}_{3}$ from 5 position), 52.7 ( $\mathrm{CH}_{3}$ from 6 position), $103.4\left(\mathrm{C}_{5}\right), 117.0$ $\left(C_{3}\right), 126.2\left(C_{6}\right), 126.3\left(C_{7}\right), 128.7\left(C_{11}\right), 128.8\left(C_{4}\right), 130.8\left(C_{10}\right), 131.0$ $\left(C_{42}\right), 136.2\left(C_{12}\right), 139.7\left(C_{9}\right), 144.7\left(C_{2}\right), 162.7$ (CO from 5 position), 164.5 (CO from 6 position), $184.2\left(\mathrm{C}_{8}\right.$, keto).

42.6. Dimethyl 7-(4-methylbenzoyl)pyrrolol1,2-b Jpyridazine-5,6-dicarboxylate (2f). A mixture of cycloimmonium salt if $(1.47 \mathrm{~g}$, $5 \mathrm{mmol}$ ) and dimethyl acetylenedicarboxylate (0.68 $\mathrm{mL}, 5.5 \mathrm{mmol})$ was suspended in anhydrous benzene, $40 \mathrm{~mL}$ under classical heating or $10 \mathrm{~mL}$ under $\mathrm{MW}$ irradiation. Then, triethylamine $(0.77 \mathrm{~mL}$, $5.5 \mathrm{mmol}$ ) was added. Under classical conditions, the solution was refluxed (oil bath) for $2 \mathrm{~h}$. Under microwave heating, the solution was exposed to microwave for $5 \mathrm{~min}$. Using MW irradiation, the best results were obtained using a constant irradiation power $(20 \% \mathrm{from}$ the full power of the magnetron, $800 \mathrm{~W}$ ) and varying the temperature (the so-called 'power control'). The resulting mixture was filtered hot to remove triethylamine hydrobromide and the clear solution was evaporated in vacuo to give the crude product, which was purified by flash chromatography $\left(99 / 1 \mathrm{CH}_{2} \mathrm{Cl}_{2} / \mathrm{CH}_{3} \mathrm{OH}\right)$ to give the dimethyl 7-(4methylbenzoyl pyrrolo 1,2-b]pyridazine-5,6-dicarboxylate $2 \mathrm{f}(0.21 \mathrm{~g}$, $12 \%$ (under classical heating) and $0.21 \mathrm{~g}, 12 \%$ (under microwaves)) as a yellow solid, mp $177-178^{\circ} \mathrm{C}$. Found: $\mathrm{C}_{1} 64.76 ; \mathrm{H}, 4.57 ; \mathrm{N}, 7.89$. $\mathrm{C}_{19} \mathrm{H}_{16} \mathrm{~N}_{2} \mathrm{O}_{5}(352)$ requires $\mathrm{C}, 64.77 ; \mathrm{H}, 4.58 ; \mathrm{N}, 7.95 \% ; R_{f}\left(99 / 1 \mathrm{CH}_{2} \mathrm{Cl}_{2}\right.$ l $\left.\mathrm{CH}_{3} \mathrm{OH}\right) 0.27$; IR ( $\left.\mathrm{KBr}, \mathrm{cm}^{1}\right): 3079$ (C-H arom.), 2953 (C-H aliph.), $1739,1708(C=0$ est $), 1635(C=0$ keto $), 1604,1537,1504,1452(C=C$, $\left.\mathrm{C}=\mathrm{N}), 1271,1105(\mathrm{C}-\mathrm{O}-\mathrm{C}) ;{ }^{1} \mathrm{H} \mathrm{NMR}\left(\mathrm{CDCl}_{3}, \delta, \mathrm{ppm},\right\}, \mathrm{Hz}\right): 2.42(\mathrm{~s}, 3 \mathrm{H}:$ $\mathrm{CH}_{3}$ from 12 position), 3.59 ( $\mathrm{s}, 3 \mathrm{H}: \mathrm{CH}_{3}$ from 5 position), $3.92(\mathrm{~s}, 3 \mathrm{H}$ : $\mathrm{CH}_{3}$ from 6 position), 7.05 (dd, $\left.J=4.4,9.2,1 \mathrm{H}: \mathrm{H}_{3}\right), 7.25(\mathrm{~d}, J=8.0,2 \mathrm{H}$ : $\left.\mathrm{H}_{11}\right), 7.71\left(\mathrm{~d}, J=8.0,2 \mathrm{H}: \mathrm{H}_{10}\right), 8.32\left(\mathrm{~d}, J=4.4,1 \mathrm{H}: \mathrm{H}_{4}\right), 8.60(\mathrm{~d}, J=9.2,1 \mathrm{H}:$ $\left.\mathrm{H}_{2}\right) ;{ }^{13} \mathrm{CNMR}$ (TMS, $\mathrm{CDCl}_{3}, \hat{\delta}$, ppm) $: 21.8$ ( $\mathrm{CH}_{3}$ from 12 position), 51.9 ( $\mathrm{CH}_{3}$ from 5 position), $52.5\left(\mathrm{CH}_{3}\right.$ from 6 position), $103.2\left(\mathrm{C}_{5}\right), 117.1\left(\mathrm{C}_{3}\right)$, $125.3\left(\mathrm{C}_{6}\right), 127.1\left(\mathrm{C}_{7}\right), 128.7\left(\mathrm{C}_{4}\right), 129.2\left(\mathrm{C}_{11}\right), 129.6\left(\mathrm{C}_{10}\right), 130.8\left(\mathrm{C}_{42}\right)$, $135.2\left(C_{12}\right), 144.3\left(C_{9}\right), 144.6\left(C_{2}\right), 163.0(C O$ from 5 position), $164.5(C O$ from 6 position), $185.3\left(C_{8}\right.$, keto).

4.2.7. Dimethyl 3-carbamoyl-1,10b-dihydropyrrolo[2,1-ajphthalazine-1,2-dicarboxylate (2g). A mixture of cycloimmonium salt $\mathbf{1 i}$ $(1.34 \mathrm{~g}, 5 \mathrm{mmol})$ and dimethyl acetylenedicarboxylate $(0.68 \mathrm{~mL}$, $5.5 \mathrm{mmol}$ ) was suspended in anhydrous benzene, $40 \mathrm{~mL}$ under classical heating or $10 \mathrm{~mL}$ under MW irradiation. Then, triethylamine $(0.77 \mathrm{~mL}, 5.5 \mathrm{mmol})$ was added. Under classical conditions, the solution was refluxed (oil bath) for $2 \mathrm{~h}$. Under microwave heating, the solution was exposed to microwave for $5 \mathrm{~min}$. Using MW irradiation, the best results were obtained using a constant irradiation power (20\% from the full power of the magnetron, 
$800 \mathrm{~W}$ ) and varying the temperature (the so-called 'power control'). The resulting mixture was filtered hot to remove triethylamine hydrobromide and the clear solution was evaporated in vacuo to give the crude product, which was purified by flash chromatography $\left(99 / 1 \mathrm{CH}_{2} \mathrm{Cl}_{2} / \mathrm{CH}_{3} \mathrm{OH}\right)$ to give the dimethyl 3-carbamoyl-1,10b-dihydropyrrolo 2,1 -ajphthalazine-1,2-dicarboxylate $2 \mathrm{~g}$ (0.64 $\mathrm{g}, 39 \%$ (under classical heating) and $1.05 \mathrm{~g}, 64 \%$ (under microwaves)) as a white solid, $\mathrm{mp} 192-193^{\circ} \mathrm{C}$. Found: $\mathrm{C}, 58.32 ; \mathrm{H}$, 4.53; $\mathrm{N}, 12.69$. $\mathrm{C}_{16} \mathrm{H}_{15} \mathrm{~N}_{3} \mathrm{O}_{5}$ (329) requires $\mathrm{C}, 58.36 ; \mathrm{H}, 4.59 ; \mathrm{N}$, $12.76 \% ; \mathrm{K}_{f}\left(99 / 1 \mathrm{CH}_{2} \mathrm{Cl}_{2} / \mathrm{CH}_{3} \mathrm{OH}\right) 0.23 ; \mathrm{IR}\left(\mathrm{KBr}, \mathrm{cm}^{1}\right): 3454(\mathrm{~N}-\mathrm{H}$ amide), 3074(C-H arom.), 2958 (C-H aliph.), 1721 ( $\mathrm{C}=\mathrm{O}$ est.), 1704 ( $\mathrm{C}=\mathrm{O}$ amide), 1601, 1569, $1558\left(\mathrm{C}=\mathrm{C}\right.$ arom.); ${ }^{1} \mathrm{H}$ NMR $\left(\mathrm{CDCl}_{3}, \delta\right.$, ppm,, $\mathrm{Hz}): 3.72\left(\mathrm{~s}, 3 \mathrm{H}: \mathrm{CH}_{3}\right.$ from 1 position), $3.86\left(\mathrm{~s}, 3 \mathrm{H}: \mathrm{CH}_{3}\right.$ from 2 position), 4.35 (d, $\left.J=13.2,1 \mathrm{H}: \mathrm{H}_{1}\right), 5.04\left(\mathrm{~d}, J=13.2,1 \mathrm{H}: \mathrm{H}_{10 \mathrm{~b}}\right), 6.18$ (br $s, 1 \mathrm{H}: \mathrm{NH}$ ), 6.41 (br $s, 1 \mathrm{H}: \mathrm{NH}), 7.27$ (dd, $\left.J=1.2,6.4,1 \mathrm{H}: \mathrm{H}_{10}\right), 7.39$ (m, overlapped peaks, $2 \mathrm{H}: \mathrm{H}_{8}, \mathrm{H}$ ) $, 7.48\left(\mathrm{~d}, J=7.6,1 \mathrm{H}: \mathrm{H}_{7}\right), 7.59$ (s, 1H: $\left.\mathrm{H}_{6}\right) ;{ }^{13} \mathrm{CNMR}$ (TMS, $\mathrm{CDCl}_{3}, \delta$, ppm): $51.7\left(\mathrm{CH}_{3}\right.$ from 1 position), $52.1\left(\mathrm{C}_{1}\right), 53.0\left(\mathrm{CH}_{3}\right.$ from 2 position), $61.3\left(\mathrm{C}_{10 \mathrm{~b}}\right), 123.3\left(\mathrm{C}_{8}\right), 125.0$ $\left(C_{2}\right), 125.8\left(C_{10}\right), 128.8\left(C_{9}\right), 131.0\left(C_{78}\right), 131.7\left(C_{7}\right), 142.5\left(C_{6}\right), 150.0$ $\left(C_{3}\right), 161.6$ (CO from 3 position), 164.5 (CO from 2 position), 173.1 (CO from 1 position).

4.2.8. 5,7-Di-(methoxycarbonyl)-pynolo(1,2-b]pynidazine (3a). A mixture of cycloimmonium salt $1 \mathrm{a}(1.17 \mathrm{~g}, 5 \mathrm{mmol})$ and methyl propiolate $(0.51 \mathrm{~mL}, 5.5 \mathrm{mmol})$ was suspended in anhydrous benzene, $40 \mathrm{~mL}$ under classical heating or $10 \mathrm{~mL}$ under MW irradiation. Then, triethylamine $(0.77 \mathrm{~mL}, 5.5 \mathrm{mmol})$ was added. Under classical conditions, the solution was refluxed (oil bath) for $2 \mathrm{~h}$. Under microwave heating, the solution was exposed to microwave for 5 min. Using MW irradiation, the best results were obtained using a constant irradiation power (20\% from the full power of the magnetron, $800 \mathrm{~W}$ ) and varying the temperature (the so-called 'power control'). The resulting mixture was filtered hot to remove triethylamine hydrobromide and the clear solution was evaporated in vacuo to give the crude product, which was purified by flash chromatography $\left(99 / 1 \quad \mathrm{CH}_{2} \mathrm{Cl}_{2} / \mathrm{CH}_{3} \mathrm{OH}\right)$ to give 5,7-di-(methoxycarbonyl)-pyrrolo[1,2-b]pyridazine $3 \mathrm{a}(0.35 \mathrm{~g}, 30 \%$ (under classical heating) and $0.68 \mathrm{~g}, 58 \%$ (under microwaves)) as a brown light solid, mp $147-148^{\circ} \mathrm{C}$. Found: $\mathrm{C}, 56.36 ; \mathrm{H}, 4.24 ; \mathrm{N}, 11.89 . \mathrm{C}_{11} \mathrm{H}_{10} \mathrm{~N}_{2} \mathrm{O}_{4}$ (234) requires $\mathrm{C}, 56.41 ; \mathrm{H}, 4.30 ; \mathrm{N}, 11.96 \% ; R_{f}\left(99 / 1 \mathrm{CH}_{2} \mathrm{Cl}_{2} / \mathrm{CH}_{3} \mathrm{OH}\right)$ 0.25; $\mathrm{IR}\left(\mathrm{KBr}_{\mathrm{r}} \mathrm{cm}^{1}\right)$ : 3101 (C-H arom.), 2960 (C-H aliph.), 1728, $1678(C=O$ est $), 1599,1561,1490,1444(C=C, C=N), 1234,1114$ $(\mathrm{C}-\mathrm{O}-\mathrm{C}) ;{ }^{1} \mathrm{H}$ NMR $\left(\mathrm{CDCl}_{3}, \delta\right.$, ppm, $\left.), \mathrm{Hz}\right): 3.92\left(\mathrm{~s}, 3 \mathrm{H}: \mathrm{CH}_{3}\right.$ from 5 position), 3.96 ( $\mathrm{s}, 3 \mathrm{H}: \mathrm{CH}_{3}$ from 7 position), 7.09 (dd, $J=9.2,4.4,1 \mathrm{H}$ : $\left.\mathrm{H}_{3}\right), 8.00\left(\mathrm{~s}, 1 \mathrm{H}: \mathrm{H}_{6}\right), 8.52\left(\mathrm{dd}, J=4.4,1.6,1 \mathrm{H}: \mathrm{H}_{4}\right), 8.63(\mathrm{dd}, J=9.2,1.6$, 1H: $\left.\mathrm{H}_{2}\right) ;{ }^{13} \mathrm{C} \mathrm{NMR}$ (TMS, CDCl $\left.3, \hat{\mathrm{o}}, \mathrm{ppm}\right): 51.5\left(\mathrm{CH}_{3}\right.$ from 5 position, COOMe), $51.8\left(\mathrm{CH}_{3}\right.$ from 7 position, COOMe $), 105.0\left(\mathrm{C}_{5}\right), 116.6\left(\mathrm{C}_{3}\right)$, $119.5\left(C_{4 a}\right), 122.5\left(C_{6}\right), 128.0\left(C_{2}\right), 133.1\left(C_{7}\right), 144.2\left(C_{4}\right), 159.6(C O$ from 7 position), 163.9 (CO from 5 position); MS (EI, m/z): 236 $(\mathrm{M}+2 ; 2 \%), 235(\mathrm{M}+1 ; 18 \%), 234\left(\mathrm{M}^{+}, 99.9 \%\right), 203$ (P.B.; 100\%), 176 $(18.4 \%), 145(21 \%), 116(5 \%), 88(10.52 \%)$.

4.2.9. 7-Ethoxy-5-methoxycarbonylpyrrolo[1,2-b]pyridazine (3b). A mixture of cycloimmonium salt $1 \mathrm{~b}(1.24 \mathrm{~g}, 5 \mathrm{mmol})$ and methyl propiolate $(0.51 \mathrm{~mL}, 5.5 \mathrm{mmol})$ was suspended in anhydrous benzene, $40 \mathrm{~mL}$ under classical heating or $10 \mathrm{~mL}$ under MW irradiation. Then, triethylamine ( $0.77 \mathrm{~mL}, 5.5 \mathrm{mmol})$ was added. Under classical conditions, the solution was refluxed (oil bath) for $2 \mathrm{~h}$. Under microwave heating, the solution was exposed to microwave for $5 \mathrm{~min}$. Using MW irradiation, the best results were obtained using a constant irradiation power ( $20 \%$ from the full power of the magnetron, $800 \mathrm{~W}$ ) and varying the temperature (the so-called 'power control'). The resulting mixture was filtered hot to remove triethylamine hydrobromide and the clear solution was evaporated in vacuo to give the crude product, which was purified by flash chromatography (99/1 $\mathrm{CH}_{2} \mathrm{Cl}_{2} / \mathrm{CH}_{3} \mathrm{OH}$ ) to give 7-ethoxy-5-methoxycarbonylpyrrolo[1,2-b] pyridczine $3 \mathrm{~b}(0.40 \mathrm{~g}, 32 \%$ (under classical heating) and $0.76 \mathrm{~g}, 61 \%$ (under microwaves)) as a white solid, $\mathrm{mp} 111-112^{\circ} \mathrm{C}$. Found: $\mathrm{C}, 57.96$; $\mathrm{H}, 4.84 ; \mathrm{N}, 11.15 . \mathrm{C}_{12} \mathrm{H}_{12} \mathrm{~N}_{2} \mathrm{O}_{4}(248)$ requires $\mathrm{C}, 58.06 ; \mathrm{H}, 4.87 ; \mathrm{N}$, $11.29 \% ; R_{f}\left(99 / 1 \mathrm{CH}_{2} \mathrm{Cl}_{2} / \mathrm{CH}_{3} \mathrm{OH}\right) 0.26 ; \mathrm{IR}\left(\mathrm{KBr}_{1} \mathrm{~cm}^{1}\right): 3106(\mathrm{C}-\mathrm{H}$ arom.), 2958 (C-Haliph.), 1718, 1711 (C = Oest.), 1601, 1561, 1502,1464 $(\mathrm{C}=\mathrm{C}, \mathrm{C}=\mathrm{N}), 1229,1132(\mathrm{C}-\mathrm{O}-\mathrm{C}) ;{ }^{1} \mathrm{H}$ NMR $\left(\mathrm{CDCl}_{3}, \delta, \mathrm{ppm}, J, \mathrm{~Hz}\right): 1.42$ ( $\mathrm{t}, J=7.2,3 \mathrm{H}: \mathrm{CH}_{3}$ from 7 position), 3.93 ( $\mathrm{s}, 3 \mathrm{H}: \mathrm{CH}_{3}$ from 5 position), 4.43 (q, $J=6.8,2 \mathrm{H}: \mathrm{CH}_{2}$ from 7 position), 7.09 (dd, $J=9.24,4,1 \mathrm{H}: \mathrm{H}_{3}$ ), $8.06\left(\mathrm{~s}, 1 \mathrm{H}: \mathrm{H}_{6}\right), 8.53\left(\mathrm{~d}_{1} J=3.2,1 \mathrm{H}: \mathrm{H}_{4}\right), 8.63\left(\mathrm{dd}, J=9.2,1.6,1 \mathrm{H}: \mathrm{H}_{2}\right) ;{ }^{13} \mathrm{C}$ NMR (TMS, $\mathrm{CDCl}_{3}, \delta, \mathrm{ppm}$ ): $14.4\left(\mathrm{CH}_{3}\right.$ from 7 position, $\mathrm{COOEt}$ ), 51.5 $\left(\mathrm{CH}_{3}\right.$ from 5 position, $\left.\mathrm{COOMe}\right), 60.7\left(\mathrm{CH}_{2}\right.$ from 7 position, $\left.\mathrm{COOEt}\right)$, $104.9\left(C_{5}\right), 116.5\left(C_{3}\right), 119.7\left(C_{4 a}\right), 122.4\left(C_{6}\right), 128.0\left(C_{2}\right), 133.1\left(C_{7}\right), 144.2$ $\left(\mathrm{C}_{4}\right), 159.2$ (CO from 7 position), 164.0 (CO from 5 position); MS (El, $m / z): 250(\mathrm{M}+2 ; 2.1 \%), 249(\mathrm{M}+1 ; 13.5), 248\left(\mathrm{M}^{+}, 100 \%\right), 217(23.4 \%)$, $203(90.1 \%), 189(41.1 \%), 176(73 \%), 145(66.7 \%), 117(9.9 \%), 70(3 \%)$.

4.2.10. Ethyl 7-carbamoylpyrrolo[1,2-b]pyridazine-5-carboxylate (3c). A mixture of cycloimmonium salt $1 \mathrm{c}(1.09 \mathrm{~g}, 5 \mathrm{mmol})$ and ethyl propiolate $(0.56 \mathrm{~mL}, 5.5 \mathrm{mmol})$ was suspended in anhydrous benzene, $40 \mathrm{~mL}$ under classical heating or $10 \mathrm{~mL}$ under $\mathrm{MW}$ irradiation. Then, triethylamine $(0.77 \mathrm{~mL}, 5.5 \mathrm{mmol})$ was added. Under classical conditions, the solution was refluxed (oil bath) for $2 \mathbf{h}$. Under microwave heating, the solution was exposed to microwave for $5 \mathrm{~min}$. Using MW irradiation, the best results were obtained using a constant irradiation power ( $20 \%$ from the full power of the magnetron, $800 \mathrm{~W}$ ) and varying the temperature (the so-called power control'). The resulting mixture was filtered hot to remove triethylamine hydrabromide and the clear solution was evaporated in vacuo to give the crude product, which was purified by flash chromatography $\left(99 / 1 \mathrm{CH}_{2} \mathrm{Cl}_{2} / \mathrm{CH}_{3} \mathrm{OH}\right)$ to give the ethyl 7-carbamoylpyrrolo $1,2-b$ )pyridazine-5-carboxylate $3 \mathrm{c}(0.33 \mathrm{~g}, 28 \%$ (under classical heating) and $0.66 \mathrm{~g}, 57 \%$ (under microwaves)) as a white solid, mp $207-208^{\circ} \mathrm{C}$. Found: $\mathrm{C}, 56.64 ; \mathrm{H}, 4.70 ; \mathrm{N}, 17.96$. $\mathrm{C}_{11} \mathrm{H}_{11} \mathrm{~N}_{3} \mathrm{O}_{3}(233)$ requires $\mathrm{C}_{4} 56.65 ; \mathrm{H}, 4.75 ; \mathrm{N}, 18.02 \% ; R_{f}(99 / 1$ $\left.\mathrm{CH}_{2} \mathrm{Cl}_{2} / \mathrm{CH}_{3} \mathrm{OH}\right) 0.20$; IR ( $\left.\mathrm{KBr}, \mathrm{cm}^{1}\right): 3439(\mathrm{~N}-\mathrm{H}$ amide), 3084 ( $C-H$ arom.), 2947 ( $C-H$ aliph.), 1717 ( $C=0$ est.), 1703 ( $C=0$ amide), $1600,1569,1522(\mathrm{C}=\mathrm{C}$ arom. $) ;{ }^{1} \mathrm{H}$ NMR $\left(\mathrm{CDCl}_{3}, \delta, \mathrm{ppm}, J, \mathrm{~Hz}\right)$ : $1.36\left(\mathrm{t}, J=7.2,3 \mathrm{H}: \mathrm{CH}_{3}\right.$ from 5 position), $4.33\left(\mathrm{q}, J=7.2,2 \mathrm{H}: \mathrm{CH}_{2}\right.$ from 5 position), 7.33 (dd, $\left.J=4.8,9.2,1 \mathrm{H}: \mathrm{H}_{3}\right), 7.79\left(\mathrm{~s}, 1 \mathrm{H}: \mathrm{H}_{6}\right), 7.95$ (br $\mathrm{s}$, $1 \mathrm{H}: \mathrm{NH}), 8.21$ (br s, $1 \mathrm{H}: \mathrm{NH}), 8.61\left(\mathrm{dd}, J=1.6,9.2,1 \mathrm{H}: \mathrm{H}_{4}\right), 8.68(\mathrm{dd}$, $\left.\jmath=1.6,4.8,1 \mathrm{H}: \mathrm{H}_{2}\right) ;{ }^{13} \mathrm{C} \mathrm{NMR}$ (TMS, $\left.\mathrm{CDCl}_{3}, \hat{o}, \mathrm{ppm}\right): 14.3\left(\mathrm{CH}_{3}\right.$ from 5 position), $59.9\left(\mathrm{CH}_{2}\right.$ from 5 position), $104.1\left(\mathrm{C}_{5}\right), 116.6\left(\mathrm{C}_{3}\right), 119.6$ $\left(C_{6}\right), 123.3\left(C_{7}\right), 128.3\left(C_{4}\right), 131.0\left(C_{4 a}\right), 144.3\left(C_{2}\right), 159.5$ (CO from 7 position), 162.8 (CO from 5 position).

4.2.11. Ethy! 7-(4-fluorobenzoyl)pyrrolol 1,2-blpyridazine-5-carboxylate (3d). A mixture of cycloimmonium salt $1 \mathrm{~d}(1.49 \mathrm{~g}, 5 \mathrm{mmol})$ and ethyl propiolate $(0.56 \mathrm{~mL}, 5.5 \mathrm{mmol})$ was suspended in anhydrous benzene, $40 \mathrm{~mL}$ under classical heating or $10 \mathrm{~mL}$ under $\mathrm{MW}$ irradiation. Then, triethylamine $(0.77 \mathrm{~mL}, 5.5 \mathrm{mmol})$ was added. Under classical conditions, the solution was refluxed (oil bath) for $2 \mathrm{~h}$. Under microwave heating, the solution was exposed to microwave for $5 \mathrm{~min}$. Using MW irradiation, the best results were obtained using a constant irradiation power (20\% from the full power of the magnetron, $800 \mathrm{~W}$ ) and varying the temperature (the so-called 'power control'). The resulting mixture was filtered hot to remove triethylamine hydrobromide and the clear solution was evaporated in vacuo to give the crude product, which was purified by flash chromatography ( $\left.99 / 1 \mathrm{CH}_{2} \mathrm{Cl}_{2} / \mathrm{CH}_{3} \mathrm{OH}\right)$ to give the ethyl 7-(4-fituorobenzoyl)pyrrolo[ 1,2-bjpyridazine-5-carboxylate $3 \mathrm{~d}(0.39 \mathrm{~g}, 25 \%$ (under classical heating) and $0.31 \mathrm{~g}, 20 \%$ (under microwaves)) as a white solid, $\mathrm{mp} 120-122^{\circ} \mathrm{C}$. Found: $\mathrm{C}, 65.35 ; \mathrm{H}, 4.13 ; \mathrm{N}, 8.89$. $\mathrm{C}_{17} \mathrm{H}_{13} \mathrm{FN}_{2} \mathrm{O}_{3}(312)$ requires $\mathrm{C}, 65.38 ; \mathrm{H}, 4.20 ; \mathrm{N}, 8.97 \% ; R_{f}(99 / 1$ $\left.\mathrm{CH}_{2} \mathrm{Cl}_{2} / \mathrm{CH}_{3} \mathrm{OH}\right) 0.28 ; \mathrm{IR}\left(\mathrm{KBr}, \mathrm{cm}^{1}\right): 3098(\mathrm{C}-\mathrm{H}$ arom.), $2962(\mathrm{C}-\mathrm{H}$ aliph.), $1708(\mathrm{C}=\mathrm{O}$ est.), $1647(\mathrm{C}=\mathrm{O}$ keto $), 1600,1506,1471(\mathrm{C}=\mathrm{C}$, $\mathrm{C}=\mathrm{N}), 1255,1097(\mathrm{C}-\mathrm{O}-\mathrm{C}) ;{ }^{1} \mathrm{H}$ NMR $\left(\mathrm{CDCl}_{3}, \delta, \mathrm{ppm}_{1} \mathrm{~J}, \mathrm{~Hz}\right) ; 1.41(\mathrm{t}$, 
$J=7.2,3 \mathrm{H}: \mathrm{CH}_{3}$ from 5 position $), 4.40\left(q_{1} J=7.2,2 \mathrm{H}: \mathrm{CH}_{2}\right.$ from 5 position), 7.18 (dd, $\left.J=4.4,9.2,1 \mathrm{H}: \mathrm{H}_{3}\right), 7.19\left(\mathrm{~d}, J=8.4,8.8,2 \mathrm{H}: \mathrm{H}_{11}\right)$, $7.73\left(\mathrm{~s}, 1 \mathrm{H}: \mathrm{H}_{6}\right), 7.95\left(\mathrm{dd}, J=5.6,8.4,2 \mathrm{H}: \mathrm{H}_{10}\right), 8.32(\mathrm{dd}, J=4.4,1 \mathrm{H}$ : $\left.\mathrm{H}_{4}\right), 8.68$ (dd, $J=9.2,1 \mathrm{H}: \mathrm{H}_{2}$ ); ${ }^{13} \mathrm{C}$ NMR (TMS, $\mathrm{CDCl}_{3}, \delta, \mathrm{ppm}$ ): 14.5 ( $\mathrm{CH}_{3}$ from 5 position), 60.5 ( $\mathrm{CH}_{2}$ from 5 position), $105.5\left(\mathrm{C}_{5}\right), 115.7$, $115.5\left(\mathrm{~d}, J=21, C_{11}\right), 117.7\left(C_{3}\right), 124.6\left(C_{6}\right), 126.5\left(C_{7}\right), 128.1\left(C_{4}\right), 132.1$, $132.0\left(d, J=9, C_{10}\right), 133.5\left(C_{4 a}\right), 135.2\left(C_{12}\right), 144.3\left(C_{2}\right), 163.5\left(C_{9}\right)_{1}$ 164.1 (CO from 5 position), $182.9\left(C_{8}\right.$, keto).

4.2.12. Ethyl 7-(4-chlorobenzoyl)pyrrolo[1,2-blpyridazine-5-carboxyIate (3e). A mixture of cycloimmonium salt $1 \mathrm{e}(1.57 \mathrm{~g}, 5 \mathrm{mmol})$ and ethyl propiolate $(0.56 \mathrm{~mL}, 5.5 \mathrm{mmol})$ was suspended in anhydrous benzene, $40 \mathrm{~mL}$ under classical heating or $10 \mathrm{~mL}$ under MW irradiation. Then, triethylamine $(0.77 \mathrm{~mL}, 5.5 \mathrm{mmol})$ was added. Under classical conditions, the solution was refluxed (oil bath) for $2 \mathrm{~h}$. Under microwave heating, the solution was exposed to microwave for $5 \mathrm{~min}$. Using MW irradiation, the best results were obtained using a constant irradiation power ( $20 \%$ from the full power of the magnetron, $800 \mathrm{~W}$ ) and varying the temperature (the so-called 'power control'). The resulting mixture was filtered hot to remove triethylamine hydrobromide and the clear solution was evaporated in vacuo to give the crude product, which was purified by flash chromatography $\left(99 / 1 \mathrm{CH}_{2} \mathrm{Cl}_{2} / \mathrm{CH}_{3} \mathrm{OH}\right.$ ) to give the ethyl 7-(4-chlorobenzoyl)pyrrolo[1,2-b]pyridazine-5-carboxylate $3 \mathrm{e}(1.36 \mathrm{~g}, 83 \%$ (under classical heating) and $1.43 \mathrm{~g}, 87 \%$ (under microwaves)) as a white solid, mp 109-110 ${ }^{\circ} \mathrm{C}$. Found: $\mathrm{C}, 62.09 ; \mathrm{H}, 3.91 ; \mathrm{N}, 8.43 . \mathrm{C}_{17} \mathrm{H}_{13} \mathrm{CIN}_{2} \mathrm{O}_{3}$ (329) requires $\mathrm{C}, 62.11 ; \mathrm{H}, 3.99 ; \mathrm{N}, 8.52 \% ; R_{f}\left(99 / 1 \mathrm{CH}_{2} \mathrm{Cl}_{2} / \mathrm{CH}_{3} \mathrm{OH}\right)$ 0.30; IR ( $\left.\mathrm{KBr}, \mathrm{cm}^{1}\right): 3095$ (C-H arom), 2954 (C-H aliph.), 1705 (C= $O$ est.), $1640(C=0$ keto $), 1520,1469,1428(C=C, C=N), 1241,1094$ $(\mathrm{C}-\mathrm{O}-\mathrm{C}) ;{ }^{1} \mathrm{H} \mathrm{NMR}\left(\mathrm{CDCl}_{3}, \delta, \mathrm{ppm}, J, \mathrm{~Hz}\right): 1.41\left(\mathrm{t}, J=7.2,3 \mathrm{H}: \mathrm{CH}_{3}\right.$ from 5 position), $4.40\left(\mathrm{q}, J=7.2,2 \mathrm{H}\right.$ : $\mathrm{CH}_{2}$ from 5 position), 7.17 (dd, $J=4.0$, 9.2, $\left.1 \mathrm{H}: \mathrm{H}_{3}\right), 7.49\left(\mathrm{~d}, J=8.4,2 \mathrm{H}: \mathrm{H}_{11}\right), 7.73\left(\mathrm{~s}, 1 \mathrm{H}: \mathrm{H}_{6}\right), 7.85$ (d, $J=8.4$, $\left.2 \mathrm{H}: \mathrm{H}_{10}\right), 8.54$ (d, $\left.J=4.0,1 \mathrm{H}: \mathrm{H}_{4}\right), 8.67\left(\mathrm{~d}, j=9.2,1 \mathrm{H}: \mathrm{H}_{2}\right) ;{ }^{13} \mathrm{C} N M R$ (TMS, $\left.\mathrm{CDCl}_{3}, \hat{\delta}, \mathrm{ppm}\right): 14.5$ ( $\mathrm{CH}_{3}$ from 5 position), $60.5\left(\mathrm{CH}_{2}\right.$ from 5 position), 105.5 $\left(C_{5}\right), 117.8\left(C_{3}\right), 124.8\left(C_{6}\right), 126.3\left(C_{7}\right), 128.1\left(C_{4}\right), 128.8$ $\left(C_{11}\right), 130.9\left(C_{10}\right), 133.6\left(C_{4 a}\right), 137.3\left(C_{12}\right), 138.7\left(C_{9}\right), 144.4\left(C_{2}\right), 163.4$ (CO from 5 position), $183.0\left(C_{8}\right.$, keto $)$.

4.2.13. Ethyl 7-(4-methylbenzoyl)pyrrolo/1,2-blpyridazine-5-carboxylate (3f). A mixture of cycloimmonium salt $\mathbf{1 f}(1.47 \mathrm{~g}, 5 \mathrm{mmol})$ and ethyl propiolate $(0.56 \mathrm{~mL}, 5.5 \mathrm{mmol})$ was suspended in anhydrous benzene, $40 \mathrm{~mL}$ under classical heating or $10 \mathrm{~mL}$ under MW irradiation. Then, triethylamine $(0.77 \mathrm{~mL}, 5.5 \mathrm{mmol})$ was added. Under classical conditions, the solution was refluxed (oil bath) for $2 \mathrm{~h}$. Under microwave heating, the solution was exposed to microwave for $5 \mathrm{~min}$. Using MW irradiation, the best results were obtained using a constant irradiation power ( $20 \%$ from the full power of the magnetron, $800 \mathrm{~W}$ ) and varying the temperature (the so-called 'power control'). The resulting mixture was filtered hot to remove triethylamine hydrobromide and the clear solution was evaporated in vacuo to give the crude product, which was purified by flash chromatograply $\left(99 / 1 \mathrm{CH}_{2} \mathrm{Cl}_{2} / \mathrm{CH}_{3} \mathrm{OH}\right)$ to give the ethyl 7-(4-methylbenzoyl)pyrrolo[1,2-blpyridazine-5-carboxylate $3 \mathrm{f}$ $(0.59 \mathrm{~g}, 38 \%$ (under classical heating) and $0.45 \mathrm{~g}, 29 \%$ (under microwaves) as a white solid, $m p 111-112^{\circ} \mathrm{C}$. Found: $\mathrm{C}, 70.10 ; \mathrm{H}$, $5.18 ; \mathrm{N}, 9.03 . \mathrm{C}_{18} \mathrm{H}_{1 \mathrm{G}} \mathrm{N}_{2} \mathrm{O}_{3}$ (308) requires $\mathrm{C}, 70.12 ; \mathrm{H}, 5.23 ; \mathrm{N}, 9.09 \%$; $R_{f}\left(99 / 1 \mathrm{CH}_{2} \mathrm{Cl}_{2} / \mathrm{CH}_{3} \mathrm{OH}\right) 0.30 ;$ IR $(\mathrm{KBr}, \mathrm{cm}): 3092$ (C-H arom.), 2970 ( $C-H$ aliph.), 1683 ( $C=$ O est $), 1635$ ( $C=$ o keto), 1604, 1533, $1506,1467(\mathrm{C}=\mathrm{C}, \mathrm{C}=\mathrm{N}), 1245,1091(\mathrm{C}-\mathrm{O}-\mathrm{C}) ;{ }^{1} \mathrm{H}$ NMR $\left(\mathrm{CDCl}_{3}, \hat{\delta}\right.$, $\left.\operatorname{ppm}_{1}, \mathrm{~Hz}\right): 1.41\left(\mathrm{t}, J=7.2,3 \mathrm{H}: \mathrm{CH}_{3}\right.$ from 5 position), $2.46(\mathrm{~s}, 3 \mathrm{H}$ : $\mathrm{CH}_{3}$ from 12 position), $4.39\left(\mathrm{q}, J=7.2,2 \mathrm{H}: \mathrm{CH}_{2}\right.$ from 5 position), 7.13 $\left(\mathrm{dd}, J=4.4,9.2,1 \mathrm{H}: \mathrm{H}_{3}\right), 7.31\left(\mathrm{~d}, J=8.0,2 \mathrm{H}: \mathrm{H}_{11}\right), 7.74\left(\mathrm{~s}, 1 \mathrm{H}: \mathrm{H}_{6}\right), 7.83$ $\left(\mathrm{d}, J=8.0,2 \mathrm{H}: \mathrm{H}_{10}\right), 8.52\left(\mathrm{dd}, J=4.4,1 \mathrm{H}: \mathrm{H}_{4}\right), 8.66(\mathrm{dd}, J=9.2,1 \mathrm{H}$ : $\mathrm{H}_{2}$ ); ${ }^{13} \mathrm{C} \mathrm{NMR}$ (TMS, $\left.\mathrm{CDCl}_{3}, \hat{0}, \mathrm{ppm}\right): 14.5\left(\mathrm{CH}_{3}\right.$ from 5 position), $21.7\left(\mathrm{CH}_{3}\right.$ from 12 position), $60.4\left(\mathrm{CH}_{2}\right.$ from 5 position), $105.2\left(\mathrm{C}_{5}\right)$, $117.4\left(C_{3}\right), 124.6\left(C_{6}\right), 128.0\left(C_{4}\right), 129.1\left(C_{11}\right), 129.1\left(C_{11}\right), 129.8\left(C_{10}\right)$,
$133.3\left(C_{4 a}\right), 136.3\left(C_{12}\right), 143.1\left(C_{9}\right), 144.2\left(C_{2}\right), 163.6(C 0$ from 5 position), 184.1 ( $\mathrm{C}_{8}$, keto).

42.14. Pyrrolo 2,1 -ajphthalazine-1,3-dicarboxylic acid dimethyl ester (3g). A mixture of cycloimmonium salt $1 \mathrm{~g}(1.42 \mathrm{~g}, 5 \mathrm{mmol})$ and methyl propiolate $(0.51 \mathrm{~mL}, 5.5 \mathrm{mmol})$ was suspended in anhydrous benzene, $40 \mathrm{~mL}$ under classical heating or $10 \mathrm{~mL}$ under $\mathrm{MW}$ irradiation. Then, triethylamine $(0.77 \mathrm{~mL}, 5.5 \mathrm{mmol})$ was added. Under classical conditions, the solution was refluxed (oil bath) for $2 \mathrm{~h}$. Under microwave heating, the solution was exposed to microwave for $5 \mathrm{~min}$. Using $\mathrm{MW}$ irradiation, the best results were obtained using a constant irradiation power $(20 \%$ from the full power of the magnetron, $800 \mathrm{~W}$ ) and varying the temperature (the so-called 'power control'). The resulting mixture was filtered hot to remove triethylamine hydrobromide and the clear solution was evaporated in vacuo to give the crude product, which was purified by flash chromatography $\left(99 / 1 \mathrm{CH}_{2} \mathrm{Cl}_{2} / \mathrm{CH}_{3} \mathrm{OH}\right)$ to give the pyrrolo [2,1-a]phthalazine-1,3-dicarboxylic acid dimethyl ester $3 \mathrm{~g}(0.58 \mathrm{~g}$, $41 \%$ (under classical heating) and $0.94 \mathrm{~g}, 66 \%$ (under microwaves)) as a white solid, mp $224-225^{\circ} \mathrm{C}$. Found: $\mathrm{C}, 63.32 ; \mathrm{H}, 4.19 ; \mathrm{N}, 9.72$. $\mathrm{C}_{15} \mathrm{H}_{12} \mathrm{~N}_{2} \mathrm{O}_{4}$ (284) requires $\mathrm{C}, 63.38 ; \mathrm{H}, 4.25 ; \mathrm{N}, 9.85 \% ; R_{f}(99 / 1$ $\left.\mathrm{CH}_{2} \mathrm{Cl}_{2} / \mathrm{CH}_{3} \mathrm{OH}\right) 0.34 ; \mathrm{IR}\left(\mathrm{KBr}_{3} \mathrm{~cm}^{1}\right)$ : $3082(\mathrm{C}-\mathrm{H}$ arom. $), 2951(\mathrm{C}-\mathrm{H}$ aliph.), $1720,1709(C=0$ est. $), 1600,1564,1519,1464(C=C, C=N)$, $1232,1131(\mathrm{C}-\mathrm{O}-\mathrm{C}) ;{ }^{1} \mathrm{H} \mathrm{NMR}\left(\mathrm{CDCl}_{3}, \hat{0}, \mathrm{ppm}, J, \mathrm{~Hz}\right): 3.90\left(\mathrm{~s}, 3 \mathrm{H}: \mathrm{CH}_{3}\right.$ from 1 position), $3.93\left(\mathrm{~s}, 3 \mathrm{H}: \mathrm{CH}_{3}\right.$ from 3 position), $7.90-7.85(\mathrm{~m}$, $\left.2 \mathrm{H}: \mathrm{H}_{2}, \mathrm{H}_{8}\right), 8.00\left(\mathrm{t}, J=7.6,8.0,1 \mathrm{H}: \mathrm{H}_{9}\right), 8.16\left(\mathrm{~d}, J=7.6,1 \mathrm{H}: \mathrm{H}_{7}\right), 9.07(\mathrm{~s}$, $\left.1 \mathrm{H}: \mathrm{H}_{6}\right), 9.62\left(\mathrm{~d}, J=8.4,1 \mathrm{H}: \mathrm{H}_{10}\right) ;{ }^{13} \mathrm{CNMR}\left(\mathrm{TMS}, \mathrm{CDCl}_{3}, \delta, \mathrm{ppm}\right): 51.2$ ( $\mathrm{CH}_{3}$ from 1 position), 51.4 ( $\mathrm{CH}_{3}$ from 3 position), $106.9(\mathrm{Cl}), 121.1$ $\left(C_{6 \mathrm{a}}\right), 121.2\left(\mathrm{C}_{2}\right), 125.5\left(\mathrm{C}_{10 \mathrm{a}}\right), 125.7\left(\mathrm{C}_{10}\right), 128.0\left(\mathrm{C}_{7}\right), 128.2\left(\mathrm{C}_{3}\right), 129.6$ $\left(C_{8}\right), 131.2\left(C_{10 b}\right), 132.7\left(C_{9}\right), 146.4\left(C_{6}\right), 158.4$ (Co from 3 position), 163.5 (CO from 1 position).

4.2.15. Pyrrolo[2,1-a]phthalazine-1,3-dicarboxylic acid 3-ethyl ester 1-methyl ester (3h). A mixture of cycloimmonium salt $\mathbf{1 h}$ (1.49 $\mathbf{g}$. $5 \mathrm{mmol}$ ) and methyl propiolate $(0.51 \mathrm{~mL}, 5.5 \mathrm{mmol})$ was suspended in anhydrous benzene, $40 \mathrm{~mL}$ under dassical heating or $10 \mathrm{~mL}$ under MW irradiation. Then, triethylamine $(0.77 \mathrm{~mL}, 5.5 \mathrm{mmol})$ was added. Under classical conditions, the solution was refluxed (oil bath) for $2 \mathrm{~h}$. Under microwave heating, the solution was exposed to microwave for $5 \mathrm{~min}$. Using MW irradiation, the best results were obtained using a constant irradiation power ( $20 \%$ from the full power of the magnetron, $800 \mathrm{~W}$ ) and varying the temperature (the so-called 'power control'). The resulting mixture was filtered hot to remove triethylamine hydrobromide and the clear solution was evaporated in vacuo to give the crude product, which was purified by flash chromatography $\left(99 / 1 \mathrm{CH}_{2} \mathrm{Cl}_{2} / \mathrm{CH}_{3} \mathrm{OH}\right)$ to give the pyrrolo[2,1-ajphthalazine-1,3-dicarboxylic acid 3-ethyl ester 1methyl ester $3 \mathrm{~h}(0.55 \mathrm{~g}, 37 \%$ (under classical heating) and $0.95 \mathrm{~g}$, $64 \%$ (under microwaves)) as a white solid, $m p 269-270^{\circ} \mathrm{C}$. Found: C, 64.36; $\mathrm{H}, 4.70 ; \mathrm{N}, 9.23 . \mathrm{C}_{16} \mathrm{H}_{14} \mathrm{~N}_{2} \mathrm{O}_{4}(298)$ requires $\mathrm{C}, 64.42 ; \mathrm{H}_{\text {, }}$ $4.73 ; \mathrm{N}, 9.39 \% ; R_{f}\left(99 / 1 \mathrm{CH}_{2} \mathrm{Cl}_{2} / \mathrm{CH}_{3} \mathrm{OH}\right) 0.40 ; \mathrm{IR}\left(\mathrm{KBr}, \mathrm{cm}^{1}\right): 3081$ (C--H arom.), 2954 (C-H aliph.), 1721, 1709 ( $C=$ O est.), 1602, 1565, $1540,1464(\mathrm{C}=\mathrm{C}, \mathrm{C}=\mathrm{N}), 1236,1132(\mathrm{C}-\mathrm{O}-\mathrm{C}) ;{ }^{1} \mathrm{H} \mathrm{NMR}\left(\mathrm{CDCl}_{3}, \delta\right.$, ppm, $J, \mathrm{~Hz}): 1.38\left(\mathrm{t}, J=7.2,3 \mathrm{H}: \mathrm{CH}_{3}\right.$ from 3 position), $3.92\left(\mathrm{~s}, 3 \mathrm{H}: \mathrm{CH}_{3}\right.$ from 1 position), 4.38 (q, $J=7.2,2 \mathrm{H}: \mathrm{CH}_{2}$ from 3 position), $7.88-7.82$ $\left(\mathrm{m}, 2 \mathrm{H}: \mathrm{H}_{2}, \mathrm{H}_{8}\right), 7.99\left(\mathrm{t}, J=7.6,8.0,1 \mathrm{H}: \mathrm{H}_{9}\right), 8.14\left(\mathrm{~d}, J=7.6,1 \mathrm{H}: \mathrm{H}_{7}\right)$, $9.06\left(\mathrm{~s}, 1 \mathrm{H}: \mathrm{H}_{6}\right), 9.61\left(\mathrm{~d}, J=8.4,1 \mathrm{H}: \mathrm{H}_{10}\right) ;{ }^{13} \mathrm{CNMR}\left(\mathrm{TMS}, \mathrm{CDCl}_{3}, \delta\right.$, ppm): 13.8 ( $\mathrm{CH}_{3}$ from 3 position), 51.3 ( $\mathrm{CH}_{3}$ from 1 position), 59.8 $\left(\mathrm{CH}_{2}\right.$ from 3 position $), 106.8(\mathrm{C} 1), 119.5\left(\mathrm{C}_{6 \mathrm{a}}\right), 121.0\left(\mathrm{C}_{2}\right), 122.0\left(\mathrm{C}_{10 \mathrm{a}}\right)$, $125.5\left(C_{10}\right), 125.6\left(C_{7}\right), 127.9\left(C_{3}\right), 128.4\left(C_{8}\right), 129.4\left(C_{10 b}\right), 132.6\left(C_{9}\right)$, $146.3\left(C_{6}\right), 157.8$ (CO from 3 position), 163.5 (CO from 1 position).

4.2.16. 3-Carbamoilpyrrolo[2,1-a]phthalazine-1-carboxylic acid methyl ester(3i). A mixture of cycloimmonium salt $1 \mathrm{i}(1.34 \mathrm{~g}, 5 \mathrm{mmol})$ and methyl propiolate $(0.51 \mathrm{~mL}, 5.5 \mathrm{mmol})$ was suspended in anhydrous benzene, $40 \mathrm{~mL}$ under classical heating or $10 \mathrm{~mL}$ under $\mathrm{MW}$ 
irradiation. Then, triethylamine $(0.77 \mathrm{~mL}, 5.5 \mathrm{mmol})$ was added. Under classical conditions, the solution was refluxed (oil bath) for $2 \mathrm{~h}$. Under microwave heating, the solution was exposed to microwave for $5 \mathrm{~min}$. Using $\mathrm{MW}$ irradiation, the best results were obtained using a constant irradiation power (20\% from the full power of the magnetron, $800 \mathrm{~W}$ ) and varying the temperature (the so-called 'power control'). The resulting mixture was filtered hot to remove triethylamine hydrobromide and the clear solution was evaporated in vacuo to give the crude product, which was purified by flash chromatography $\left(99 / 1 \mathrm{CH}_{2} \mathrm{Cl}_{2} / \mathrm{CH}_{3} \mathrm{OH}\right)$ to give 3-carbamollpyrrolo[2,1-a]phthalazine-1-arboxylic acid methyl ester $3 i(0.54 \mathrm{~g}$ $40 \%$ (under classical heating) and $0.92 \mathrm{~g}, 68 \%$ (under microwaves)) as a yellow solid, $\mathrm{mp} 181-182{ }^{\circ} \mathrm{C}$. Found: $\mathrm{C}, 62.39 ; \mathrm{H}, 4.12 ; \mathrm{N}, 15.57$. $\mathrm{C}_{14} \mathrm{H}_{11} \mathrm{~N}_{3} \mathrm{O}_{3}(269)$ requires $\mathrm{C}, 62.45 ; \mathrm{H}, 4.73 ; \mathrm{N}, 15.61 \% ; R_{f}(99 / 1$ $\left.\mathrm{CH}_{2} \mathrm{Cl}_{2} / \mathrm{CH}_{3} \mathrm{OH}\right) 0.17 ; \mathrm{IR}\left(\mathrm{KBr}, \mathrm{cm}^{1}\right): 3439(\mathrm{~N}-\mathrm{H}$ amide $), 3072(\mathrm{C}-\mathrm{H}$ arom.), $2954(\mathrm{C}-\mathrm{H}$ aliph.), 1714 ( $\mathrm{C}=\mathrm{O}$ est.),1704 ( $\mathrm{C}=\mathrm{O}$ amide), $1600,1564,1540,1462(\mathrm{C}=\mathrm{C}, \mathrm{C}=\mathrm{N}) ;{ }^{1} \mathrm{H} \mathrm{NMR}\left(\mathrm{CDCl}_{3}, \delta, \mathrm{ppm}, \jmath, \mathrm{Hz}\right)$ : 3.92 (s, 3H: $\mathrm{CH}_{3}$ from 1 position), 6.11 (br s, $1 \mathrm{H}: \mathrm{NH}$ ), 6.30 (br s, $1 \mathrm{H}$ : NH), $7.87-7.81\left(\mathrm{~m}, 2 \mathrm{H}: \mathrm{H}_{2}, \mathrm{H}_{8}\right), 7.99\left(\mathrm{t}, J=7.6,8.0,1 \mathrm{H}: \mathrm{Hg}_{9}, 8.14\right.$ (d, $\left.J=7.6,1 \mathrm{H}: \mathrm{H}_{7}\right), 9.06\left(\mathrm{~s}, 1 \mathrm{H}: \mathrm{H}_{6}\right), 9.60\left(\mathrm{~d}, j=8.4,1 \mathrm{H}: \mathrm{H}_{10}\right) ;{ }^{13} \mathrm{C}$ NMR (TMS, $\mathrm{CDCl}_{3}, \delta, \mathrm{ppm}$ ): 51.3 ( $\mathrm{CH}_{3}$ from 1 position), 106.5 (C1), 119.1 $\left(\mathrm{C}_{6 \mathrm{a}}\right), 120.8\left(\mathrm{C}_{2}\right), 121.4\left(\mathrm{C}_{10 \mathrm{a}}\right), 125.6\left(\mathrm{C}_{10}\right), 125.7\left(\mathrm{C}_{7}\right), 127.8\left(\mathrm{C}_{3}\right), 128.3$ $\left(C_{8}\right), 129.4\left(C_{10 b}\right), 132.8\left(C_{9}\right), 146.1\left(C_{6}\right), 159.2$ (CO from 3 position), 163.4 (CO from 1 position).

4.2.17. 5-Cyano-4a,5,6,7-tetrahydropymolo[1,2-b]pyridazine-7-carboxamide ( $4 b$ ). A mixture of cycloimmonium salt $1 c(1.09 \mathrm{~g}, 5 \mathrm{mmol}$ ) and acrylonitrile $(0.36 \mathrm{~mL}, 5.5 \mathrm{mmol})$ was suspended in anlyydrous benzene, $40 \mathrm{~mL}$ under classical heating or $10 \mathrm{~mL}$ under MW irradiation. Then, triethylamine $(0.77 \mathrm{~mL}, 5.5 \mathrm{mmol})$ was added. Under classical conditions, the solution was refluxed (oil bath) for $3 \mathrm{~h}$. Under microwave heating, the solution was exposed to microwave for $5 \mathrm{~min}$. Using MW irradiation, the best results were obtained using a constant irradiation power ( $20 \%$ from the full power of the magnetron, $800 \mathrm{~W}$ ) and varying the temperature (the so-called 'power control'). The resulting mixture was filtered hot to remove triethylamine hydrobromide and the clear solution was evaporated in vacuo to give the crude product, which was purified by flash chromatography (99/1 $\mathrm{CH}_{2} \mathrm{Cl}_{2} / \mathrm{CH}_{3} \mathrm{OH}$ ) to give 5-cyano-4a,5,6,7-tetrahydropymolo[1,2-b]pyridazine-7-carboxamide $4 \mathrm{~b}(0.29 \mathrm{~g}, 31 \%$ (under classical heating) and $0.49 \mathrm{~g}, 52 \%$ (under microwaves)) as a white solid, mp $158-159^{\circ} \mathrm{C}$. Found: $\mathrm{C}, 56.80 ; \mathrm{H}, 5.22 ; \mathrm{N}, 29.37 . \mathrm{Cg}_{9} \mathrm{H}_{10} \mathrm{~N}_{4} \mathrm{O}$ (190) requires $\mathrm{C}, 56.83 ; \mathrm{H}$, $5.30 ; \mathrm{N}, 29.46 \% ; R_{f}\left(99 / 1 \mathrm{CH}_{2} \mathrm{Cl}_{2} / \mathrm{CH}_{3} \mathrm{OH}\right) 0.22 ; \mathrm{IR}\left(\mathrm{KBr}, \mathrm{cm}^{1}\right): 3451$ (N-H amide), 3077 (C-H arom), 2952 (C-H aliph.), $2168(\mathrm{CN}), 1706$ (C=O amide), $1602,1566,1533$ (C=C arom.); ${ }^{1} \mathrm{H} \mathrm{NMR}\left(\mathrm{CDCl}_{3}, \hat{0}, \mathrm{ppm}_{4}\right.$ $J, \mathrm{~Hz}): 2.07\left(\mathrm{~h}, \mathrm{~J}=3.2,4.4,13.8,1 \mathrm{H}: \mathrm{H}_{6 \mathrm{a}}\right), 2.23(\mathrm{~h}, J=8.4,10.0,13.8,1 \mathrm{H}$ : $\left.H_{6 b}\right), 3.44\left(h, j=4.4,6.4,10.0,1 H: H_{5}\right), 4.20\left(d_{1} J=2.8,6.4,1 H: H_{4 a}\right), 4.37$ (dd, $\left.J=3.2,8.4,1 \mathrm{H}: \mathrm{H}_{7}\right), 6.07-6.06$ ( $\mathrm{m}$, overlapped peaks, $2 \mathrm{H}: \mathrm{H}_{3}, \mathrm{H}_{4}$ ), $6.71\left(\mathrm{t}, J=2.4,4.8,1 \mathrm{H}: \mathrm{H}_{2}\right), 7.18$ (br $\left.s, 1 \mathrm{H}: \mathrm{NH}\right), 7.52$ (br $\left.s, 1 \mathrm{H}: \mathrm{NH}\right) ;{ }^{13} \mathrm{C}$ NMR (TMS, $\left.\mathrm{CDCl}_{3}, \delta, \mathrm{ppm}\right): 29.2\left(\mathrm{C}_{6}\right), 34.0\left(\mathrm{C}_{5}\right), 56.4\left(\mathrm{C}_{4 \mathrm{a}}\right), 67.7\left(\mathrm{C}_{7}\right)$, $119.3\left(\mathrm{C}_{4}\right), 121.1(\mathrm{CN}), 124.2\left(\mathrm{C}_{3}\right), 134.0\left(\mathrm{C}_{2}\right), 172.9$ (CO from 7 position).

4.2.18. 1-Cyano-1,2,3,10b-tetrahydropyrolo[2,1-a]phthalazine-3carboxamide (4c). A mixture of cycloimmonium salt $11(1.34 \mathrm{~g}$ $5 \mathrm{mmol})$ and acrylonitrile $(0.36 \mathrm{~mL}, 5.5 \mathrm{mmol})$ was suspended in anhydrous benzene, $40 \mathrm{~mL}$ under classical heating or $10 \mathrm{~mL}$ under MW irradiation. Then, triethylamine $\left(0.77 \mathrm{~mL}_{*} 5.5 \mathrm{mmol}\right)$ was added. Under classical conditions, the solution was refluxed (oil bath) for $3 \mathrm{~h}$. Under microwave heating, the solution was exposed to microwave for $5 \mathrm{~min}$. Using MW imadiation, the best results were obtained using a constant irradiation power ( $20 \%$ from the full power of the magnetron, $800 \mathrm{~W}$ ) and varying the temperature (the so-called 'power control'). The resulting mixture was filtered hot to remove triethylamine hydrobromide and the clear solution was evaporated in vacuo to give the crude product, which was purified by flash chromatography $\left(99 / 1 \mathrm{CH}_{2} \mathrm{Cl}_{2} / \mathrm{CH}_{3} \mathrm{OH}\right)$ to give 1 -cyano-1,2,3,10b- tetrahydropymrolol2,1-a]phthalazine-3-carboxamide $4 \mathrm{c}(0.35 \mathrm{~g}, 29 \%$ (under classical heating) and $0.64 \mathrm{~g}, 53 \%$ (under microwaves)) as a white solid, $\mathrm{mp} 203-204^{\circ} \mathrm{C}$. Found: $\mathrm{C}, 65.00 ; \mathrm{H}, 4.94 ; \mathrm{N}, 23.26$. $\mathrm{C}_{13} \mathrm{H}_{12} \mathrm{~N}_{4} \mathrm{O}(240)$ requires $\mathrm{C}_{1} 64.99 ; \mathrm{H}, 5.03 ; \mathrm{N}, 23.32 \% ; R_{f}(99 / 1$ $\left.\mathrm{CH}_{2} \mathrm{Cl}_{2} / \mathrm{CH}_{3} \mathrm{OH}\right) 0.23 ; \mathrm{IR}$ (KBr, $\left.\mathrm{Cm}^{1}{ }^{1}\right): 3446(\mathrm{~N}-\mathrm{H}$ amide), $3082(\mathrm{C}-\mathrm{H}$ arom), $2963(\mathrm{C}-\mathrm{H}$ aliph.), $2171(\mathrm{CN}), 1708$ ( $=0$ amide), 1600, 1575, $1554(\mathrm{C}=\mathrm{C}$ arom. $) ;{ }^{1} \mathrm{H}$ NMR $\left(\mathrm{CDCl}_{3}, \delta, \mathrm{ppm}, \mathrm{J}, \mathrm{Hz}\right): 2.46(\mathrm{~h}, \mathrm{j}=3.6,4.4$, $\left.12.8,1 \mathrm{H}: \mathrm{H}_{2 \mathrm{a}}\right), 2.82\left(\mathrm{~h}_{\mathrm{z}} \mathrm{j}=9.2,9.6,12.8,1 \mathrm{H}: \mathrm{H}_{2 \mathrm{~b}}\right), 3.29(\mathrm{~h}, J=3.4,6.8$, 9.6, $\left.1 \mathrm{H}: \mathrm{H}_{1}\right), 4.55$ (d, $\left.J=6.8,1 \mathrm{H}: \mathrm{H}_{10 \mathrm{~b}}\right), 4.81$ (dd, $J=4.4,9.2,1 \mathrm{H}: \mathrm{H}_{3}$ ) 5.52 (br s, $1 \mathrm{H}: \mathrm{NH}$ ), 6.83 (br s, $1 \mathrm{H}: \mathrm{NH}), 7.18$ (d, $\left.J=7.2,1 \mathrm{H}: \mathrm{H}_{10}\right), 7.34$ $\left(\mathrm{dd}, \mathrm{J}=8.4,1 \mathrm{H}: \mathrm{H}_{7}\right), 7.49-7.45\left(\mathrm{~m}\right.$, overlapped peaks, $\left.3 \mathrm{H}: \mathrm{H}_{6}, \mathrm{H}_{8}, \mathrm{H}_{9}\right)$; ${ }^{13} \mathrm{C} \mathrm{NMR}\left(\mathrm{TMS}, \mathrm{CDCl}_{3}, \delta, \mathrm{ppm}\right): 29.8\left(\mathrm{C}_{2}\right), 35.9\left(\mathrm{C}_{1}\right), 59.7\left(\mathrm{C}_{10 \mathrm{~b}}\right), 69.9$ $\left(\mathrm{C}_{3}\right), 121.2(\mathrm{CN}), 126.6\left(\mathrm{C}_{7}\right), 126.6\left(\mathrm{C}_{10}\right), 129.8\left(\mathrm{C}_{8}\right), 131.8\left(\mathrm{C}_{9}\right), 140.0$ $\left(\mathrm{C}_{6}\right), 173.1$ (CO from 3 position).

4.2.19. 6,7-Dihydro-5,7-di-(methoxycarbonyl)-pyrrolo[1,2-b/pyridazine (5a). A mixture of cycloimmonium salt $1 \mathrm{a}(1.17 \mathrm{~g}, 5 \mathrm{mmol}$ ) and methyl acrylate $(0.50 \mathrm{~mL}, 5.5 \mathrm{mmol})$ was suspended in anhydrous benzene, $40 \mathrm{~mL}$ under classical heating or $10 \mathrm{~mL}$ under $\mathrm{MW}$ irradiation. Then, triethylamine $(0.77 \mathrm{~mL}, 5.5 \mathrm{mmol})$ was added. Under classical conditions, the solution was refluxed (oil bath) for $3 \mathrm{~h}$. Under microwave heating, the solution was exposed to microwave for $5 \mathrm{~min}$. Using MW irradiation, the best results were obtained using a constant irradiation power ( $20 \%$ from the full power of the magnetron, $800 \mathrm{~W}$ ) and varying the temperature (the so-called 'power control'). The resulting mixture was filtered hot to remove triethylamine hydrobromide and the clear solution was evaporated in vacuo to give the crude product, which was purified by flash chromatography $\left(99 / 1 \mathrm{CH}_{2} \mathrm{Cl}_{2} / \mathrm{CH}_{3} \mathrm{OH}\right)$ to give 6,7 -dihydro5,7-di-(methoxycarbonyl)-pyrrolo[1,2-b]pyridazine $\mathbf{5 a}(0.27 \mathrm{~g}, 23 \%$ (under classical heating) and $0.57 \mathrm{~g}, 48 \%$ (under microwaves)) as a ruby-red solid, $\mathrm{mp} 109-110^{\circ} \mathrm{C}$. Found: $\mathrm{C}, 55.81 ; \mathrm{H}, 5.05 ; \mathrm{N}, 11.76$. $\mathrm{C}_{11} \mathrm{H}_{12} \mathrm{~N}_{2} \mathrm{O}_{4}(236)$ requires $\mathrm{C}, 55.93 ; \mathrm{H}, 5.12 ; \mathrm{N}, 11.86 \% ; R_{f}(99 / 1$ $\left.\mathrm{CH}_{2} \mathrm{Cl}_{2} / \mathrm{CH}_{3} \mathrm{OH}\right) 0.39$; $\mathrm{RR}\left(\mathrm{KBr}_{7} \mathrm{~cm}{ }^{1}\right): 3103(\mathrm{C}-\mathrm{H}$ arom.), $2958(\mathrm{C}-\mathrm{H}$ aliph. $), 1732,1635(\mathrm{C}=\mathrm{O}$ est $), 1600,1558,1502,1469(\mathrm{C}=\mathrm{C}, \mathrm{C}=\mathrm{N})$, $1223,1117(\mathrm{C}-\mathrm{O}-\mathrm{C}) ;{ }^{1} \mathrm{H}$ NMR $\left(\mathrm{CDCl}_{3}, \delta, \mathrm{ppm}, J, \mathrm{~Hz}\right): 2.95\left(\mathrm{dd}_{1} J=16.1\right.$, $\left.6.4,1 \mathrm{H}: \mathrm{H}_{6 \mathrm{~b}}\right), 3.25\left(\mathrm{t}, J=16.1,12.2,1 \mathrm{H}: \mathrm{H}_{6 \mathrm{a}}\right), 3.80\left(\mathrm{~s}, 3 \mathrm{H}: \mathrm{CH}_{3}\right.$ from 7 position), 3.96 ( $\mathrm{s}, 3 \mathrm{H}: \mathrm{CH}_{3}$ from 5 position), 4.91 (q, $J=12.2,6.4,1 \mathrm{H}$ : $\left.\mathrm{H}_{7}\right), 6.53\left(\mathrm{dd}, J=9.2,4.8,1 \mathrm{H}: \mathrm{H}_{3}\right), 7.24\left(\mathrm{dd}, J=4.8,1.6,1 \mathrm{H}: \mathrm{H}_{4}\right), 7.59(\mathrm{~d}$, $\left.j=9.2,1 \mathrm{H}: \mathrm{H}_{2}\right) ;{ }^{13} \mathrm{C} \mathrm{NMR}\left(\mathrm{TMS}, \mathrm{CDCl}_{3}, \delta, \mathrm{ppm}\right): 30.0\left(\mathrm{C}_{6}\right), 51.2\left(\mathrm{CH}_{3}\right.$ from 5 position, $\mathrm{COOMe})_{2} 52.9\left(\mathrm{CH}_{3}\right.$ from 7 position, $\left.\mathrm{COOMe}\right), 65.7$ $\left(C_{7}\right), 118.8\left(C_{5}\right), 126.1\left(C_{3}\right), 126.9\left(C_{4}\right), 136.7\left(C_{2}\right), 151.3\left(C_{42}\right), 163.5$ (CO from 5 position), 169.2 (CO from 7 position).

4.2.20. 6,7-Dihydro-7-ethoxy-5-methoxycarbonylpyrrolo 1,2-b]pyridazine (5b). A mixture of cycloimmonium salt $1 \mathrm{~b}(1.24 \mathrm{~g}, 5 \mathrm{mmol})$ and methyl acrylate $(0.50 \mathrm{~mL}, 5.5 \mathrm{mmol})$ was suspended in anhydrous benzene, $40 \mathrm{~mL}$ under classical heating or $10 \mathrm{~mL}$ under $\mathrm{MW}$ irradiation. Then, triethylamine $(0.77 \mathrm{~mL}, 5.5 \mathrm{mmol})$ was added. Under classical conditions, the solution was refluxed (oil bath) for $3 \mathrm{~h}$. Under microwave heating, the solution was exposed to microwave for $5 \mathrm{~min}$. Using MW irradiation, the best results were obtained using a constant irradiation power $(20 \%$ from the full power of the magnetron, $800 \mathrm{~W}$ ) and varying the temperature (the so-called 'power control'). The resulting mixture was filtered hot to remove triethylamine hydrobromide and the clear solution was evaporated in vacuo to give the crude product, which was purified by flash chromatography $\left(99 / 1 \mathrm{CH}_{2} \mathrm{Cl}_{2} / \mathrm{CH}_{3} \mathrm{OH}\right)$ to give 6,7 -dihydro7-ethoxy-5-methoxycarbonylpyrrolo[ 1,2-b]pyridazine $5 \mathrm{~b}(0.29 \mathrm{~g}, 23 \%$ (under classical heating) and $0.61 \mathrm{~g}, 49 \%$ (under microwaves)) as a ruby-red solid, $\mathrm{mp} 102-103^{\circ} \mathrm{C}$. Found: $\mathrm{C}, 57.50 ; \mathrm{H}, 5.59 ; \mathrm{N}, 11.03$. $\mathrm{C}_{12} \mathrm{H}_{14} \mathrm{~N}_{2} \mathrm{O}_{4}$ (250) requires $\mathrm{C}, 57.59 ; \mathrm{H}, 5.64 ; \mathrm{N}, 11.19 \% ; R_{f}(99 / 1$ $\left.\mathrm{CH}_{2} \mathrm{Cl}_{2} / \mathrm{CH}_{3} \mathrm{OH}\right)$ 0.39; IR $\left(\mathrm{KBr}, \mathrm{cm}^{1}\right): 3103$ (C-H arom.), $2925(\mathrm{C}-\mathrm{H}$ aliph. $), 1735,1637(C=0$ est $), 1597,1558,1460,1433(C=C, C=N)$,

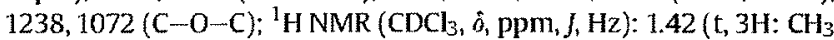
from 7 position), $2.94\left(\mathrm{dd}, J=15.6,6.4,1 \mathrm{H}: \mathrm{H}_{6 \mathrm{~b}}\right), 3.26(\mathrm{t}, J=15.6,13.6$, 
1H: $\mathrm{H}_{6 \mathrm{a}}$ ) 4.20 (s, 3H: $\mathrm{CH}_{3}$ from 5 position), $4.42\left(\mathrm{q}, 2 \mathrm{H}: \mathrm{CH}_{2}\right.$ from 7 position), $4.89\left(\mathrm{dd}, J=13.6,6.4,1 \mathrm{H}: \mathrm{H}_{7}\right), 6.53\left(\mathrm{dd}, J=9.2,3.6,1 \mathrm{H}: \mathrm{H}_{3}\right)$ $7.24\left(\mathrm{dd}_{\imath} J=3.6,1.2,1 \mathrm{H}: \mathrm{H}_{4}\right), 7.59\left(\mathrm{~d}, J=9.2,1 \mathrm{H}: \mathrm{H}_{2}\right) ;{ }^{13} \mathrm{C}$ NMR (TMS, $\left.\mathrm{CDCl}_{3}, \hat{\delta}, \mathrm{ppm}\right): 14.0\left(\mathrm{CH}_{3}\right.$ from 7 position, COOEt $), 30.4\left(\mathrm{C}_{6}\right), 50.6$ ( $\mathrm{CH}_{3}$ from 5 position, COOMe), $59.9\left(\mathrm{CH}_{2}\right.$ from 7 position, COOEt), $66.2\left(C_{7}\right), 118.8\left(C_{5}\right), 126.0\left(C_{3}\right), 126.1\left(C_{4}\right), 136.6\left(C_{2}\right), 151.3\left(C_{4 a}\right)$ 167.0 ( $\mathrm{CO}$ from 5 position), 167.8 ( $\mathrm{CO}$ from 7 position).

4.2.21. 5-Cyano-6,7-dihydro-7-methoxycarbonylpyrrolo[ 1,2-b]pyridazine $(5 \mathrm{c})$. A mixture of cycloimmonium salt $1 \mathrm{a}(1.17 \mathrm{~g}, 5 \mathrm{mmol})$ and acrylonitrile $(0.36 \mathrm{~mL}, 5.5 \mathrm{mmol})$ was suspended in anhydrous benzene, $40 \mathrm{~mL}$ under classical heating or $10 \mathrm{~mL}$ under MW irradiation. Then, triethylamine $(0.77 \mathrm{~mL}, 5.5 \mathrm{mmol})$ was added. Under classical conditions, the solution was refluxed (oil bath) for $3 \mathrm{~h}$. Under microwave heating, the solution was exposed to microwave for $5 \mathrm{~min}$. Using MW irradiation, the best results were obtained using a constant irradiation power (20\% from the full power of the magnetron, $800 \mathrm{~W}$ ) and varying the temperature (the so-called 'power control'). The resulting mixture was filtered hot to remove triethylamine hydrobromide and the clear solution was evaporated in vacuo to give the crude product, which was purified by flash chromatography $\left(99 / 1 \mathrm{CH}_{2} \mathrm{Cl}_{2} / \mathrm{CH}_{3} \mathrm{OH}\right)$ to give 5-cyano-6,7-dihydro7-methoxycarbonylpyrrolol 1,2-bjpyridazine $5 \mathrm{c}(0.37 \mathrm{~g}, 36 \%$ (under classical heating) and $0.63 \mathrm{~g}, 62 \%$ (under microwaves)) as a mauve solid, mp 107-108 ${ }^{\circ} \mathrm{C}$. Found: $\mathrm{C}, 59.14 ; \mathrm{H}, 4.36 ; \mathrm{N}, 20.60 . \mathrm{C}_{10} \mathrm{H}_{9} \mathrm{~N}_{3} \mathrm{O}_{2}$ (203) requires $\mathrm{C}, 59.11 ; \mathrm{H}, 4.46 ; \mathrm{N}, 20.68 \% ; R_{f}\left(99 / 1 \mathrm{CH}_{2} \mathrm{Cl}_{2} / \mathrm{CH}_{3} \mathrm{OH}\right)$ $0.39 ; \mathrm{IR}\left(\mathrm{KBr}, \mathrm{cm}^{1}\right.$ ): 3102 (C-H arom.), 2960 (C-H aliph.), 2179 $(\mathrm{CN}), 1744(\mathrm{C}=\mathrm{O}$ est $), 1598,1558,1496,1461(\mathrm{C}=\mathrm{C}, \mathrm{C}=\mathrm{N}), 1231$, $1134(\mathrm{C}-\mathrm{O}-\mathrm{C}) ;{ }^{1} \mathrm{H} \mathrm{NMR}\left(\mathrm{CDCl}_{3}, \delta, \mathrm{ppm}, J, \mathrm{~Hz}\right): 2.92(\mathrm{dd}, j=15.8,6.8$, $\left.1 \mathrm{H}: \mathrm{H}_{6 \mathrm{~b}}\right), 3.25\left(\mathrm{t}, J=15.8,12.2,1 \mathrm{H}: \mathrm{H}_{63}\right), 3.82\left(\mathrm{~s}, 3 \mathrm{H}: \mathrm{CH}_{3}\right.$ from 7 position), 4.90 (q, $\left.J=12.2,6.8,1 \mathrm{H}: \mathrm{H}_{7}\right), 6.49$ (dd, $J=9.2,4.2,1 \mathrm{H}: \mathrm{H}_{3}$ ), $6.88\left(\mathrm{dd}, J=4.2,1.6,1 \mathrm{H}: \mathrm{H}_{4}\right), 7.20$ (br s, $\left.1 \mathrm{H}: \mathrm{H}_{2}\right) ;{ }^{13} \mathrm{C}$ NMR (TMS $\left.\mathrm{CDCl}_{3}, \delta, \mathrm{ppm}\right): 31.1\left(\mathrm{C}_{6}\right), 53.1\left(\mathrm{CH}_{3}\right.$ from 7 position $), 65.3\left(\mathrm{C}_{7}\right), 118.8$ $(\mathrm{CN}), 118.8\left(\mathrm{C}_{5}\right), 124.7\left(\mathrm{C}_{4}\right), 126.1\left(\mathrm{C}_{3}\right), 136.8\left(\mathrm{C}_{2}\right), 152.2\left(\mathrm{C}_{4 a}\right), 169.9$ (CO from 7 position).

4.2.22. 5-Cyano-6,7-dihydro-7-ethoxycarbonylpyrrolo[1,2-b]pyridazine (5d). A mixture of cycloimmonitum salt $1 \mathrm{~b}(1.24 \mathrm{~g}, 5 \mathrm{mmol})$ and acrylonitrile $(0.36 \mathrm{~mL}, 5.5 \mathrm{mmol})$ was suspended in anhydrous benzene, $40 \mathrm{~mL}$ under classical heating or $10 \mathrm{~mL}$ under MW irradiation. Then, triethylamine ( $0.77 \mathrm{ml}, 5.5 \mathrm{mmol})$ was added. Under classical conditions, the solution was refluxed (oil bath) for $3 \mathrm{~h}$. Under microwave heating, the solution was exposed to microwave for $5 \mathrm{~min}$. Using MW irradiation, the best results were obtained using a constant irradiation power (20\% from the full power of the magnetron, $800 \mathrm{~W}$ ) and varying the temperature (the so-called 'power control'). The resulting mixture was filtered hot to remove triethylamine hydrobromide and the clear solution was evaporated in vacuo to give the crude product, which was purified by flash chromatography $\left(99 / 1 \mathrm{CH}_{2} \mathrm{Cl}_{2} / \mathrm{CH}_{3} \mathrm{OH}\right)$ to give 5-cyano-6,7-dihydro7-ethoxycarbonylpyrrolo[1,2-bJpyridazine $5 \mathrm{~d}(0.42 \mathrm{~g}, 39 \%$ (under classical heating) and $0.71 \mathrm{~g}, 65 \%$ (under microwaves)) as a mauve solid, mp $110-111^{\circ} \mathrm{C}$. Found: $\mathrm{C}, 60.79 ; \mathrm{H}, 5.05 ; \mathrm{N}, 19.21 . \mathrm{C}_{11} \mathrm{H}_{11} \mathrm{~N}_{3} \mathrm{O}_{2}$ (217) requires $\mathrm{C}, 60.82 ; \mathrm{H}, 5.10 ; \mathrm{N}, 19.34 \% ; R_{f}\left(99 / 1 \mathrm{CH}_{2} \mathrm{Cl}_{2} / \mathrm{CH}_{3} \mathrm{OH}\right)$ 0.38; IR ( $\left.\mathrm{KBr}, \mathrm{cm}^{1}\right): 3080$ (C-H arom.), 2920 (C-H aliph.), 2173 $(\mathrm{CN}), 1743(\mathrm{C}=\mathrm{O}$ est. $), 1603,1560,1506,1469(\mathrm{C}=\mathrm{C}, \mathrm{C}=\mathrm{N}), 1199$ $1031(\mathrm{C}-\mathrm{O}-\mathrm{C}):{ }^{1} \mathrm{H} \mathrm{NMR}\left(\mathrm{CDCl}_{3}, \delta, \mathrm{ppm}, J, \mathrm{~Hz}\right): 1.32\left(\mathrm{t}, 3 \mathrm{H}: \mathrm{CH}_{3}\right.$ from 7 position $), 2.91\left(\mathrm{dd}, J=14.8,6.8,1 \mathrm{H}: \mathrm{H}_{6 \mathrm{~b}}\right), 3.25(\mathrm{dd}, J=14.8,13.6,1 \mathrm{H}$ : $\mathrm{H}-6 \mathrm{a}), 4.27$ (q, $2 \mathrm{H}$ : $\mathrm{CH}_{2}$ from 7 position), $4.88(\mathrm{dd}, J=13.6,6.8,1 \mathrm{H}$ : $\left.\mathrm{H}_{7}\right), 6.50$ (dd, $\left.J=9.2,4.2,1 \mathrm{H}: \mathrm{H}_{3}\right), 6.91\left(\mathrm{~d}, J=9.2,1 \mathrm{H}: \mathrm{H}_{4}\right), 7.21(\mathrm{~s}, 1 \mathrm{H}$ : $\left.\mathrm{H}_{2}\right):{ }^{13} \mathrm{C}$ NMR (TMS, $\left.\mathrm{CDCl}_{3}, \delta, \mathrm{ppm}\right): 14.1$ ( $\mathrm{CH}_{3}$ from 7 position), 31.1 $\left(\mathrm{C}_{6}\right), 62.3\left(\mathrm{CH}_{2}\right.$ from 7 position $), 65.5\left(\mathrm{C}_{7}\right), 118.9\left(\mathrm{C}_{5}\right), 119.1(\mathrm{CN})$ $124.7\left(C_{4}\right), 126.2\left(C_{3}\right), 136.9\left(C_{2}\right), 152.3\left(C_{4 a}\right), 169.9$ (CO from 7 position); MS (EI, $m / z): 219(\mathrm{M}+2,1 \%), 218(11 \%), 217\left(\mathrm{M}^{+}, 77 \%\right), 172$ (5\%), 144 (P.B., 100\%), $117(50 \%), 89(19 \%), 63(14 \%)$.

\section{Acknowledgements}

To CNCSIS Bucuresti, grants no. $1155 / 2006$ and grant N.A.T.O. Program Security Through Science no. PDD(CP)-(CBP.EAP.CLC 982499)/20.11.2006, for financial support.

\section{References and notes}

1. Valeur, B. Molecular Fluorescence; Wiley-VCH: Weinheim, 2002

2. (a) Kido, J.; Okamoto, Y. Chem. Rev. 2002, 102, 2357-2368; (b) Lee, S.; Nakamura, I; Tutsui, T. Org. Lett. 2001, 3, 2005-2007.

3. Thompson, M. A.; Forrest, S. R. Nature 2000, 403, 750-751.

4. Mcouade, D. T.; Pullen, A. E.; Swager, T. M. Chem. Rev 2000, 100, 2537-2574

5. Rotaru, A. V; Druta, I. I.; Deser, T.; Muller, T. J. Helv. Chim. Acta 2005, 88 $1798-1812$.

6. (a) Vasilescu, $M_{\text {; }}$ Bandula, R; Cramariuc, O.; Hukka, T.; Lemmetyinen, $H$.; Rantala, T.; Dumitrascu, F. J. Photochem. Photobiol, A 2008, 194, 308-317; (b) Swamy, K. M. K.; Park, M. S.; Han, S. J.; Kim, S. K.; Kim J. H.; Lee, C; Bang, H. Kim, Y.; Kina, S J. Yoona, J Tetrahedion 2005, 61, 10227-10234; (c) Mitstimori, T.; Craig, I. M.; Martini, I. B.; Schwartz, B. J.; Wudl, F. Macromolecuies 2005, 38, 4698-4704; (d) Mitsumori, T.; Bendikov, M.; Sedo, J; Wudl, F. Chem Mater. 2003, 15, 3759-3768; (e) Cheng, Y.; Ma, B.; Wudl F. J. Mater. Chem. 1999, 9 $2183-2188$.

7. (a) Zbancioc, G. N.; Mangalagiu, 1. 1. Tetrahedron 2010, 66, 278-282; (b) Zbancioc, G. N.; Mangalagiu, I. I. Synlett $2006,804-806$

8. Chen, C. H.; Shi, J. Coord. Chem. Rev. 1998, 171, 161-167.

9. Friend, R. H.; Gymer, R. W.; Holmes, A. B; Burroughes, J. H.; Marks, R. N.; Taliani, C.; Bradley, D. D.; Santos, D. A.; Bredas, J. L; Logdlund, M.; Salaneck, W. R. Nature 1999, 397, 121-123.

10. (a) Kappe, O. C.; Dallinger, D.; Murphree, S. S. Proctical Microwave Synthesis for Organic Chemists; Wiley-VCH: Weinheim, Germany, 2009; (b) Loupy, A. Microwaves in Organic Synthesis; Wiley-VCH: Weinheim, Germany, 2006: (c) Kappe, $O$. $C$; Stadler, A. Microwaves in Organic and Medicinal Chemistry; WileyKappe, O. C; Stadler, A. Microwaves it
VCH: Weinheim, Germany, 2005.

11. (a) Kappe, O. C. Angew. Chem., Int Ed. 2004, 43, 6250-6284; (b) Perreux, L. Loupy, A. Tetrahedron 2001, 57, 9199-9223.

12. (a) Butnatiu, R; Mangalagit, I, I. Bioorg. Met. Chem 2009, 17, 2823-2879; (b) Dima, St.; Zbancioc, G.; Mangalagiu, 1. I. J. Serb. Chim 5oc. 2006, 71, 103-110; (c) Dima, St.; Zbancioc, G.; Mangalagiu, 1. L.J. Serb. Chim Soc. 2006, 7., 103-110; (c) 2005, 50, 353-358; (d) Zbancioc, G.; Caprosu, M.; Moldoveanu, C.; Mangalagí, 1. 1. Arkivoc 2005, 5, 174-187; (e) Dima, St; Mangalagiu, I. I.; Caprosu, M.; Georgescu, M.; Petrovanu, M. Rev. Roum. Chim. 2000, 45, 555-560

13. Parker C A Photoluminescence of Solusions; Elsevier: Amsterdam, 1968. 